\title{
An annotated checklist of the macrofungi (Ascomycota, Basidiomycota, and Glomeromycota) from Quintana Roo, Mexico
}

\author{
Javier Isaac de la Fuente ${ }^{1,2}$, Jesús García-Jiménez ${ }^{3}$, Caribell Yuridia López ${ }^{4}$, Iván Oros-Ortega ${ }^{5}$, \\ Reyna Yazuly Vela-Hernández ${ }^{5}$, Gonzalo Guevara-Guerrero ${ }^{3}$, Fortunato Garza-Ocañas ${ }^{6}$,

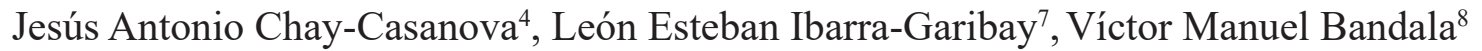

1 Universidad de Quintana Roo, División de Ciencias de la Salud, Av. Erick Paolo Martínez S/N, Chetumal, Quintana Roo, CP 77039, México. 2 Instituto Wozniak, Calzada del centenario \#590, CP 77019, Colonia del bosque, Chetumal, Quintana Roo, México. 3 Tecnológico Nacional de México. Instituto Tecnológico de Ciudad Victoria, Boulevard Emilio Portes Gil \#1301, Cd Victoria, Tamaulipas, CP 87010, México. 4 Tecnológico Nacional de México, Instituto Tecnológico de Chetumal, Avenida Insurgentes \# 330, Col. David G. Gutiérrez, Chetumal, Quintana Roo, CP 77013, México. 5 Tecnológico Nacional de México, Instituto Tecnológico de la Zona Maya, Carretera Chetumal-Escácerga, km 21.5, Juan Sarabia, Quintana Roo, CP 77965, México. 6 Universidad Autónoma de Nuevo León, Campus Linares, Facultad de Ciencias Forestales, Carretera Nacional km 145, Linares, Nuevo León, AP 41 67700, México. 7 Ecología de Artrópodos en Ambientes Extremos, Unidad Multidisciplinaria de Docencia e Investigación, Facultad de Ciencias, Universidad Nacional Autónoma de México campus Juriquilla, Boulevard Juriquilla 3001, Querétaro, Querétaro, CP 76230, México. 8 Instituto de Ecología, Carretera antigua a Coatepec 351, El Haya, Xalapa 91070, Veracruz, México.

Corresponding author: Jesús García-Jiménez, jgarjim@yahoo.com.mx

\begin{abstract}
According to records available in the literature, 380 species of fungi and fungus-like organisms are known for the state of Quintana Roo on the Yucatán Peninsula of Mexico. We carried out mycological surveys in this state, following the classical methods for collecting, describing, and curating macrofungi. As a result of our research, 21 species are recorded for the first time for Quintana Roo, and Amanita arenicola O. K. Milller \& Lodge, Inocybe xerophytica Pegler, and Russula cremeolilacina Pegler are recorded for the first time for Mexico. The distinctive characteristics and photographs are provided for the new records. As a result of this study, 401 species of macrofungi are known for Quintana Roo.
\end{abstract}

Keywords

Diversity, funga, macrofungi, tropical fungi, Yucatán Peninsula.

Academic editor: Meike Piepenbring | Received 9 February 2020 | Accepted 23 March 2020 | Published 26 May 2020

Citation: de la Fuente JI, García-Jiménez J, López CY, Oros-Ortega I, Vela-Hernández RY, Guevara-Guerrero G, Garza-Ocañas F, Chay-Casanova JA, Ibarra-Garibay LE, Bandala-Muñoz VM (2020) An annotated checklist of the macrofungi (Ascomycota, Basidiomycota, and Glomeromycota) from Quintana Roo, Mexico. Check List 16 (3): 627-648. https://doi.org/10.15560/16.3.627

\section{Introduction}

Mexico harbors a great fungal diversity, and its wide variety of geographical conditions, orography, and habitat promotes niches where fungal diversity thrives (Aguirre-Acosta et al. 2014). Guzmán (1998) estimated that 200,000 fungal species might occur in Mexico but less than $10 \%$ have been studied. So far, most fungal research has been focused on fungi occurring in temperate forests. Until 2008, only 2135 species had been 
recorded for Mexico mainly from temperate forest (Aguirre-Acosta et al. 2014). More than 900 species were recorded from tropical forest but studies regarding tropical fungi are still scarce (Guzmán et al. 1997).

The Mexican states of Quintana Roo, Campeche, and Yucatan, as well as a part of Belize and Guatemala in Central America make up the floristic province called Yucatan Peninsula. It is characterized by its deciduous, sub-deciduous, or evergreen forests. In the state of Quintana Roo, most of the arboreal species of the Yucatan Peninsula are represented, so the mycobiota of Quintana Roo is expected to be representative of the Yucatan Peninsula (Ibarra-Manríquez et al. 1995; Valdéz-Hernández and Islebe 2011).

The state of Quintana Roo has an interesting fungal diversity, which has been studied since the late 19th century by Millspaug (Chío and Guzmán 1983). The studies of Chío and Guzmán (1982), Guzmán-Dávalos and Guzmán (1982), Guzmán (1983), San Martín and Rogers (1988), Pérez-Silva et al. (1992), Ryvarden and Guzmán (1993), San Martín et al. (1995), San Martín et al. (1999), Guzmán (2003), and Pompa-González et al. (2011) have contributed knowledge on fungi for Quintana Roo by presenting the first checklist of macrofungi and describing several new species. As a result of those studies, 405 species of fungi and fungus like organisms (including lichenized fungi and myxomycetes) have been reported from the state of Quintana Roo (López et al. 2011).

Deforestation and land-use changes due to agricultural practices and new tourist developments are some of the main challenges for conservation of the fungal biodiversity in tropical forest in Quintana Roo (Valdéz-Hernández and Islebe 2011). Furthermore, loss of forest coverage may lead many fungal species to extinction, and some of them have not even been previously recorded nor described. On the other hand, there are taxonomic obstacles for correct identification, a paucity of trained mycologists, and few publications (Mueller and Schmit 2007). Furthermore, some forests, such as the forests in flooded lowlands and the pine savanna, have many interesting fungal species that are not yet formally recorded. The aim of this paper is to summarize and update information on current knowledge of macrofungi diversity from the forests of the state of Quintana Roo. To address this situation, we present our new data from our mycological studies, and we compile from the literature lists of species of fungal species collected in various ecosystems in Quintana Roo, Mexico.

\section{Methods}

Study area. The state of Quintana Roo, where collections were made (Fig 1) is located in southeastern Mexican, on the Yucatan Peninsula. The most representative forests are evergreen tropical forest composed mainly of Brosimum alicastrum Sw., Bursera simaruba (L.) Sarg., Manilkara zapota (L.) van Royen, Metopium brownei (Jacq.) Urb., and Vitex gaumeri Greenm. Lowland forest present Cameraria latifolia L., Gymnopodium floribundum Rolfe, Haematoxylum campechianum L., $M$. brownei, and Terminalia buceras (L.) C. Wright; coastal vegetation composed of Chrysobalanus icaco L., Coccoloba uvifera (L.) L., Cordia sebestena L., and Pouteria campechiana (Kunth) Baehni; pine savanna composed of Pinus caribaea Morelet, H. campechianum, Byrsonima crassifolia (L.) Kunth, Curetella america L. and Crescentia cujete L.; and secondary vegetación composed of Cecropia peltata L., Lysiloma latisiliquum (L.) Benth. and Piscidia piscipula (L.) Sarg., among numerous other species (Macario-Sánchez and Sánchez 2011; Valdéz-Hernández and Islebe 2011).

Fieldwork. Sampling and collection of macrofungi specimens follow the methods presented by Lodge et al. (2004). Hand-cut sections were mounted in $\mathrm{KOH} \mathrm{5 \%}$ (potassium hydroxide) and Meltzer reagent in order to make microscopic observations of the species. The specimens were identified using specialized literature such as Pegler (1983), Webber and Smith (1985), Guzmán (2003), and other publications cited below. The specimens were deposited in the "José Castillo Tovar" herbarium at the Instituto Tecnológico de Ciudad Victoria (ITCV) and the mycological collection of the Instituto Tecnológico de la Zona Maya (ITZM).

Literature review. The literature-based checklist was based on specialized published literature to local, country, and world levels that cite macrofungi from Quintana Roo, which was found by consulting ISI Web of Knowledge, ResearchGate, and the UNITE database for molecular identification of fungi (https://unite.ut.ee/). Searches were made using the key words: Quintana Roo + basidiomes; Quintana Roo + macrofungi; Quintana Roo + Ascomycetes, and Yucatán Peninsula + Macrofungi. Published sources in journals, book chapters, scientific notes, and scientific books were considered for the check list; theses and congress results were not considered. Accepted names are according to Index Fungorum (http://www.indexfungorum.org/names/names.asp). The species are systematically classified according to Kirk et al. (2008).

Table 1. Collecting sites in Quintana Roo, Mexico.

\begin{tabular}{|c|c|c|c|}
\hline Localities & $\begin{array}{l}\text { Geographic } \\
\text { coordinates }\end{array}$ & Vegetation type & $\begin{array}{l}\text { Elevation } \\
\text { a.s.l. (m) }\end{array}$ \\
\hline 0xtankáh & $18^{\circ} 36^{\prime} \mathrm{N}, 088^{\circ} 13^{\prime} \mathrm{W}$ & $\begin{array}{l}\text { Secondary vegetation and } \\
\text { evergreen tropical forest }\end{array}$ & 14 \\
\hline $\begin{array}{l}\text { Hermenegildo } \\
\text { Galeana }\end{array}$ & $18^{\circ} 10^{\prime} \mathrm{N}, 089^{\circ} 14^{\prime} \mathrm{W}$ & $\begin{array}{l}\text { Evergreen tropical forest and } \\
\text { lowland forest }\end{array}$ & 229 \\
\hline Blasillo & $18^{\circ} 09^{\prime} \mathrm{N}, 088^{\circ} 13^{\prime} \mathrm{W}$ & Lowland forest & 213 \\
\hline Laguna Guerrero & $18^{\circ} 45^{\prime} \mathrm{N}, 088^{\circ} 11^{\prime} \mathrm{W}$ & Lowland forest & 9 \\
\hline Jaguactal & $18^{\circ} 14^{\prime} \mathrm{N}, 088^{\circ} 57^{\prime} \mathrm{W}$ & Lowland forest and pine savanna & 132 \\
\hline Chetumal & $18^{\circ} 31^{\prime} \mathrm{N}, 088^{\circ} 18^{\prime} \mathrm{W}$ & $\begin{array}{l}\text { Secondary vegetation and } \\
\text { coastal vegetation }\end{array}$ & 8 \\
\hline Road to El Huasteco & $19^{\circ} 18^{\prime} \mathrm{N}, 088^{\circ} 16^{\prime} \mathrm{W}$ & Evergreen tropical forest & 33 \\
\hline San Pedro Peralta & $18^{\circ} 42^{\prime} \mathrm{N}, 088^{\circ} 49^{\prime} \mathrm{W}$ & Secondary vegetation & 175 \\
\hline Kohunlich & $18^{\circ} 25^{\prime} \mathrm{N}, 088^{\circ} 47^{\prime} \mathrm{W}$ & Secondary vegetation & 120 \\
\hline Bacalar & $18^{\circ} 45^{\prime} \mathrm{N}, 088^{\circ} 20^{\prime} \mathrm{W}$ & Secondary vegetation & 19 \\
\hline
\end{tabular}



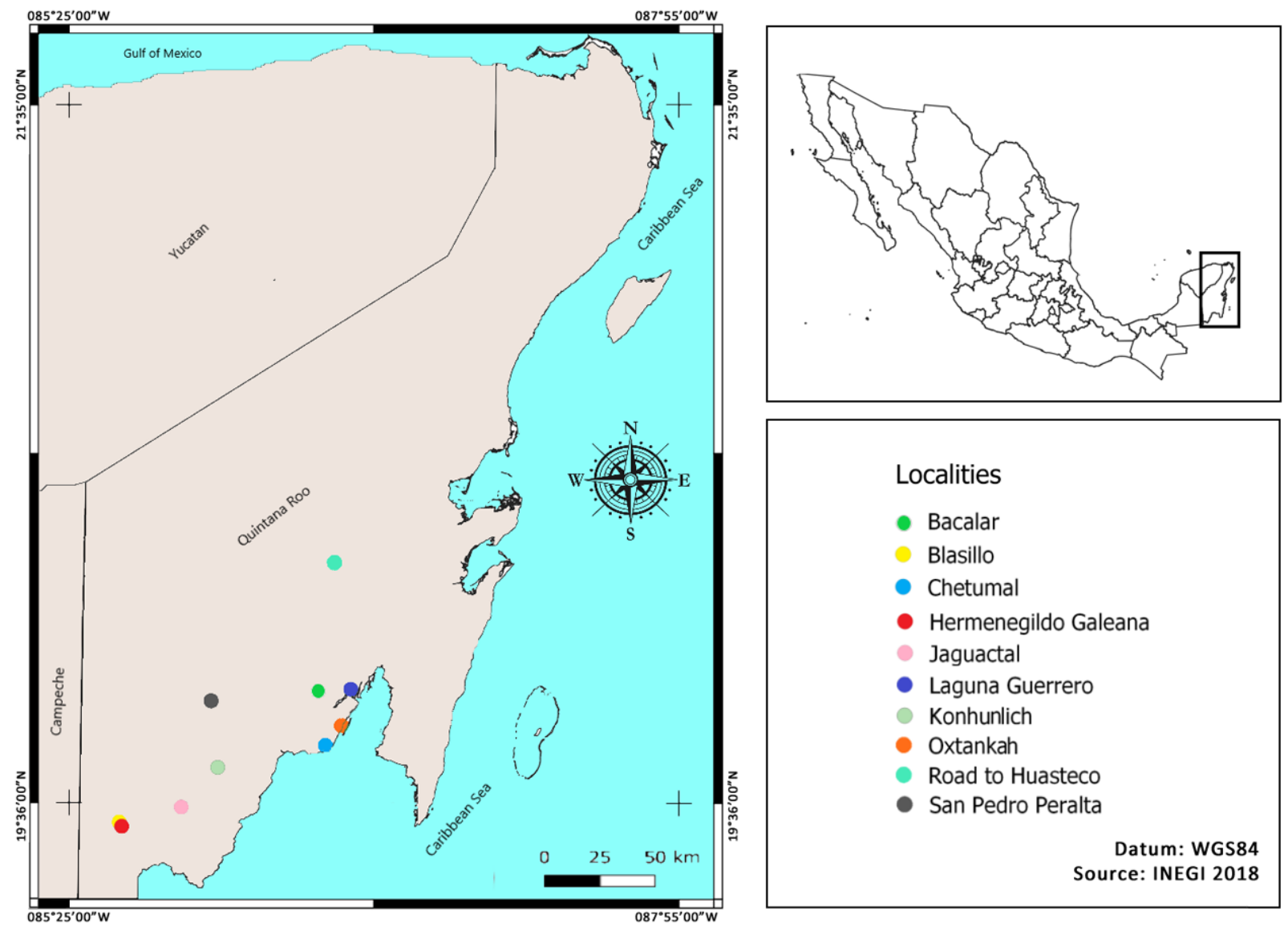

Figure 1. Collecting sites in Quintana Roo, Mexico.

\section{Results}

Among specimens of macrofungi that we collected in Quintana Roo, 77 species of macrofungi were identified. Among these, 21 species are new records for Quintana Roo (Figs 2, 3) and Amanita arenicola O. K. Miller \& Lodge, Inocybe xerophytica Pegler, and Russula cremeolilacina Pegler are new records for Mexico. Most of the species belong to Basidiomycota (65 species), followed by Ascomycota (11 species) and Glomeromycota (1 species). Our analysis of literature records showed that there are 380 species previously known from Quintana Roo. The total list, 401 species, was checked and taxonomic names were updated according to Index Fungorum (http://www.indexfungorum.org/names/names. asp). Species newly recorded from Quintana Roo are marked with an asterisk (*). Most of the species (317 species) belong to Basidiomycota, followed by Ascomycota (83 species) and Glomeromycota (1 species). The most representative families are Xylariaceae (44 species), Polyporaceae (41 species), and Agaricaceae (30 species) (Table 2).

\section{Checklist of macrofungi from Quintana Roo, Mexico}

Species names marked with an asterisk (*) are reported for the first time for Mexico. They are described in detail below. For photographs of selected species, see Figures $2-5$.

\section{Ascomycota \\ Coronophorales \\ Nitschkiaceae \\ Calyculosphaeria calyculus (Mont.) Fitzp. \\ Literature: Medel et al. (1999).}

\section{Hypocreales}

\section{Ophiocordycipitaceae}

*Ophiocordyceps melolonthae (Tul. \& C. Tul.) G.H.

Sung, J.M. Sung, Hywel-Jones \& Spatafora. Fig. 2A.

\section{Nectriaceae}

Nectria aurantiaca (Tul. \& C. Tul.) Jacz.

Literature: Guzmán (2003).

Table 2. Most common taxa of macrofungi known from Quintana Roo, Mexico, ranked according to numbers of species reported per family.

\begin{tabular}{lllcc}
\hline Phylum & Order & Family & Genera & Species \\
\hline Ascomycota & Xylariales & Xylariaceae & 4 & 44 \\
Basidiomycota & Polyporales & Polyporaceae & 14 & 41 \\
Basidiomycota & Agaricales & Agaricaceae & 11 & 30 \\
Ascomycota & Xylariales & Hypoxylaceae & 5 & 28 \\
Basidiomycota & Hymenochaetales & Hymenochaetaceae & 10 & 24 \\
Basidiomycota & Agaricales & Marasmiaceae & 11 & 22 \\
Basidiomycota & Agaricales & Entolomataceae & 1 & 13 \\
Basidiomycota & Agaricales & Psathyrellaceae & 3 & 12 \\
Basidiomycota & Polyporales & Ganodermataceae & 3 & 11 \\
Basidiomycota & Agaricales & Geastraceae & 2 & 10 \\
Basidiomycota & Agaricales & Tricholomataceae & 8 & 9 \\
\hline
\end{tabular}




\section{Hysteriales}

\section{Hysteriaceae}

Hysterium angustatum Alb. \& Schwein. Literature: Guzmán (1983).

\section{Pezizales}

\section{Sarcoscyphaceae}

Cookeina speciosa (Fr.) Dennis. Literature: Guzmán (2003).

Cookeina sulcipes (Berk.) Kuntze. Literature: Guzmán (1983).

Cookeina tricholoma (Mont.) Kuntze. Literature: Guzmán (1983), Guzmán (2003). Fig. 4A

*Geodina guanacastensis Denison.

Fig. 2B.

Phillipsia crispata (Berk. \& M.A. Curtis).

Literature: Tun-Cano (2018).

Phillipsia domingensis (Berk.) Berk. ex Denison

Literature: Guzmán (1983), Guzmán (2003). Fig. 4B.

Phillipsia hartmannii (W. Phillips) Rifai.

Literature: Guzmán (1983), Medel et al. (1999).

\section{Xylariales}

\section{Hypoxylaceae}

Annulohypoxylon archeri (Berk.)Y.M. Ju, J.D. Rogers \& H.M. Hsieh.

Literature: Medel et al. (1999), Medel (2002).

Annulohypoxylon moriforme (Henn.)Y.M. Ju, J.D. Rogers \& H.M. Hsieh.

Literature: Medel et al. (1999), San Martín et al. (1999). Annulohypoxylon pseudostipitatum (Y.M. Ju \& J.D.

Rogers) Y.M. Ju, J.D. Rogers \& H.M. Hsieh.

Literature: Medel et al. (1999), San Martín et al. (1999).

Annulohypoxylon stygium (Lév.)Y.M. Ju, J.D. Rogers \&

H.M. Hsieh.

Literature: San Martín et al. (1999).

Annulohypoxylon truncatum (Starbäck) Y.M. Ju, J.D.

Rogers \& H.M. Hsieh.

Literature: Medel et al. (1999), San Martín et al. (1999), Medel (2002).

Daldinia brachysperma F. San Martín, Y.M. Ju \& J.D. Rogers.

Literature: Ju et al. (1997).

Daldinia concentrica (Bolton) Ces. \& De Not. Literature: Guzmán (1983), Pompa-Gonzáles et al. (2011).

Daldinia eschscholtzii (Ehrenb.) Rehm. Literature: Guzmán (1983), Ju et al. (1997), Medel (2002), Pompa-Gonzáles et al. (2011). Fig. 4C.

Hypoxylon aeruginosum J.H. Mill.

Literature: Medel et al. (1999), San Martín et al. (1999).

Hypoxylon crocopeplum Berk. \& M.A. Curtis.

Literature: Medel et al. (1999), San Martín et al. (1999), Medel (2002).

Hypoxylon dieckmannii Theiss.

Literature: San Martín et al. (1999).

Hypoxylon duranii J.D. Rogers.

Literature: San Martín et al. (1999).
Hypoxylon erythrostroma J.H. Mill.

Literature: San Martín et al. (1999).

Hypoxylon fendleri Berk.ex Cooke.

Literature: Medel et al. (1999), San Martín et al. (1999).

Hypoxylon haematostroma Mont.

Literature: Guzmán (1983), San Martín et al. (1999),

Medel (2002).

Hypoxylon hypomiltum Mont.

Literature: Guzmán (1983), Ju and Rogers (1997),

Medel et al. (1999), Medel (2002).

Hypoxylon lenormandii Berk. \& M.A. Curtis.

Literature: San Martín et al. (1999).

Hypoxylon lividipigmentum F. San Martín, Y.M. Ju \& J.D. Rogers.

Literature: Medel et al. (1999), San Martín et al. (1999).

Hypoxylon macrocarpum Pouzar.

Literature: San Martín et al. (1999).

Hypoxylon monticulosum Mont.

Literature: Medel et al. (1999), San Martín (1999).

Hypoxylon polyporus (Starbäck) Y.M. Ju \& J.D. Rogers.

Literature: San Martín et al. (1999).

Hypoxylon rubiginosum (Pers.) Fr.

Literature: Guzmán (1983), Medel (2002).

Hypoxylon sublimbatum (Durieu \& Mont.) P.M.D. Martin.

Literature: Medel et al. (1999).

Hypoxylon subrutilum Starbäck.

Literature: San Martín et al. (1999).

Phylacia globosa Lév.

Literature: Guzmán (1983), Pompa-Gonzáles et al. (2011).

Phylacia poculiformis (Mont.) Mont.

Literature: Chay-Casanova and Medel (2000),

Guzmán (2003), Pompa-Gonzáles et al. (2011).

Phylacia turbinata (Berk.) Dennis.

Literature: Guzmán (1983), Chay-Casanova and Medel (2000).

Thuemenella cubispora (Ellis \& Holw.) Boedijn. Literature: Medel (2001).

\section{Xylariaceae}

Biscogniauxia capnodes (Berk.)Y.M. Ju \& J.D. Rogers. Literature: Medel et al. (1999).

Biscogniauxia nummularia (Bull.) Kuntze.

Literature: Pérez-Silva et al. (1992).

Kretzschmaria cetrarioides (Welw. \& Curr.) Sacc. Literature: San Martín and Rogers (1993).

Poronia oedipus (Mont.) Mont. Literature: Guzmán (2003).

Xylaria allantoidea (Berk.) Fr.

Literature: San Martín and Rogers (1989), San

Martín et al. (1995), Medel et al. (1999).

Xylaria anisopleura (Mont.) Fr.

Literature: San Martín and Rogers (1989), San

Martín et al. (1995), Medel et al. (1999).

Xylaria arbuscula Sacc.

Literature: San Martín and Rogers (1989), San

Martín et al. (1995). 

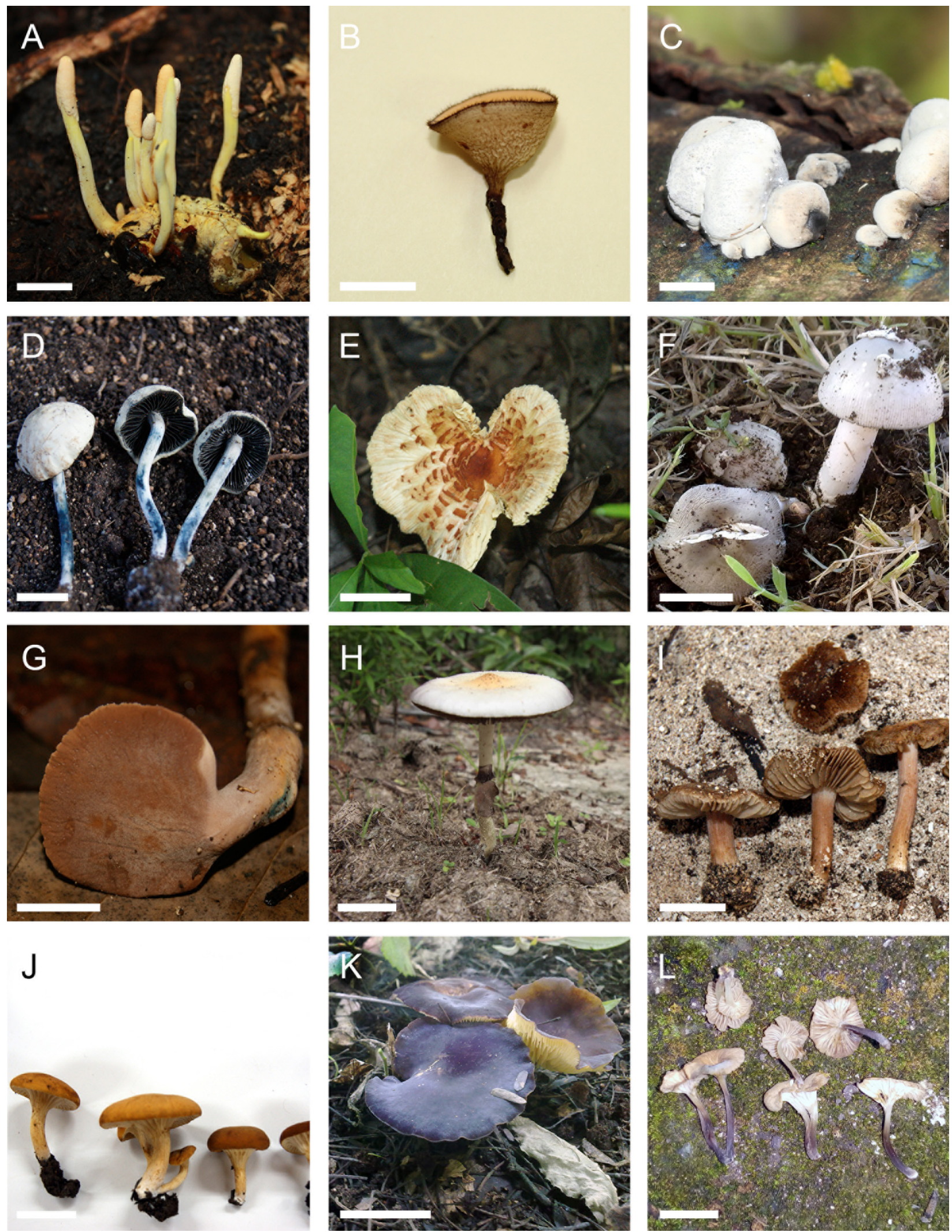

Figure 2. New records from Quintana Roo. A. Ophiocordyceps melolonthae. B. Geodina guanacastensis. C. Xylaria mesenterica. D. Paneolus cyanescens. E. Rugosospora ochraceobadia. F. Amanita arenicola. G. Pseudofistulina radicata. H. Psilocybe cubensis. I. Inocybe xerophytica. J. Neopaxillus dominicanus. K. Calocybe cyanea. L. Gerronema bryogeton. Scale bars $=10 \mathrm{~mm}$.

Xylaria bambooensis Lloyd.

Literature: San Martín and Rogers (1989), Medel et al. (1999), Guzmán (2003).

Xylaria brachiata Sacc.
Literature: San Martín et al. (1995), Medel et al. (1999).

Xylaria coccophora Mont.

Literature: San Martín and Rogers (1989), 

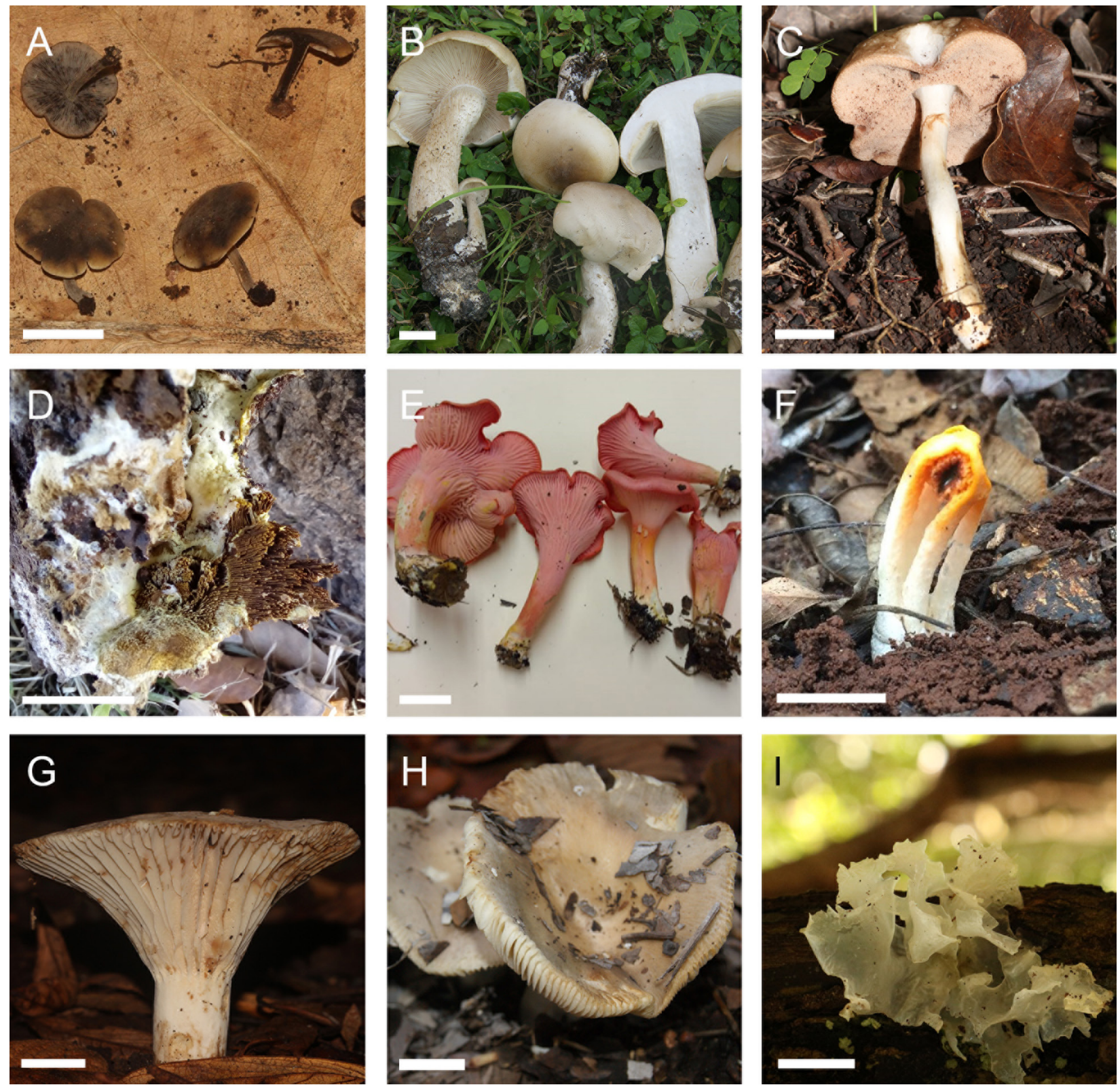

Figure 3. New records from Quintana Roo. A. Hydropus nigrita. B. Macrocybe titans. C. Mucilopilus mexicanus. D. Gyrodontium sacchari. E. Cantharellus coccolobae. F. Laternea dringii. G. Lactifluus nebulosus. H. Russula cremeolilacina. J. Tremella fusiformis. Scale bars $=10 \mathrm{~mm}$.

Pérez-Silva et al. (1992), San Martín et al. (1995), Medel et al. (1999), Guzmán (2003).

Xylaria corniformis (Fr.) Fr.

Literature: San Martín et al. (1995).

Xylaria cubensis (Mont.) Fr.

Literature: San Martín and Rogers (1989), Guzmán (1983), San Martín et al. (1995).

Xylaria delicatula Starbäck.

Literature: San Martín et al. (1995), Medel et al. (1999).

Xylaria dichotoma (Mont.) Mont.

Literature: San Martín et al. (1995), Medel et al. (1999).

Xylaria feejeensis (Berk.) Fr.

Literature: San Martín et al. (1995).

Xylaria fockei (Miq.) Cooke.

Literature: Guzmán (1983).

Xylaria gracillima (Fr.) Fr.

Literature: San Martín and Rogers (1989), San
Martín et al. (1995), Medel et al. (1999).

Xylaria guianensis (Mont.) Fr.

Literature: Chío and Guzmán (1982), Chay-Casanova and Medel (2000).

Xylaria hypoxylon (L.) Grev.

Literature: Guzmán (1983), San Martín et al.

(1995),Medel et al. (1999), Pompa-Gonzáles et al. (2011).

Xylaria ianthinovelutina (Mont.) Fr.

Literature: San Martín and Rogers (1989), Pérez-

Silva et al. (1992), San Martín et al. (1995), Medel et al. (1999).

Xylaria inaequalis Berk. \& M.A. Curtis.

Literature: San Martín and Rogers (1989), San

Martín et al. (1995), Medel et al. (1999).

Xylaria juniperus Starbäck.

Literature: San Martín et al. (1995). 

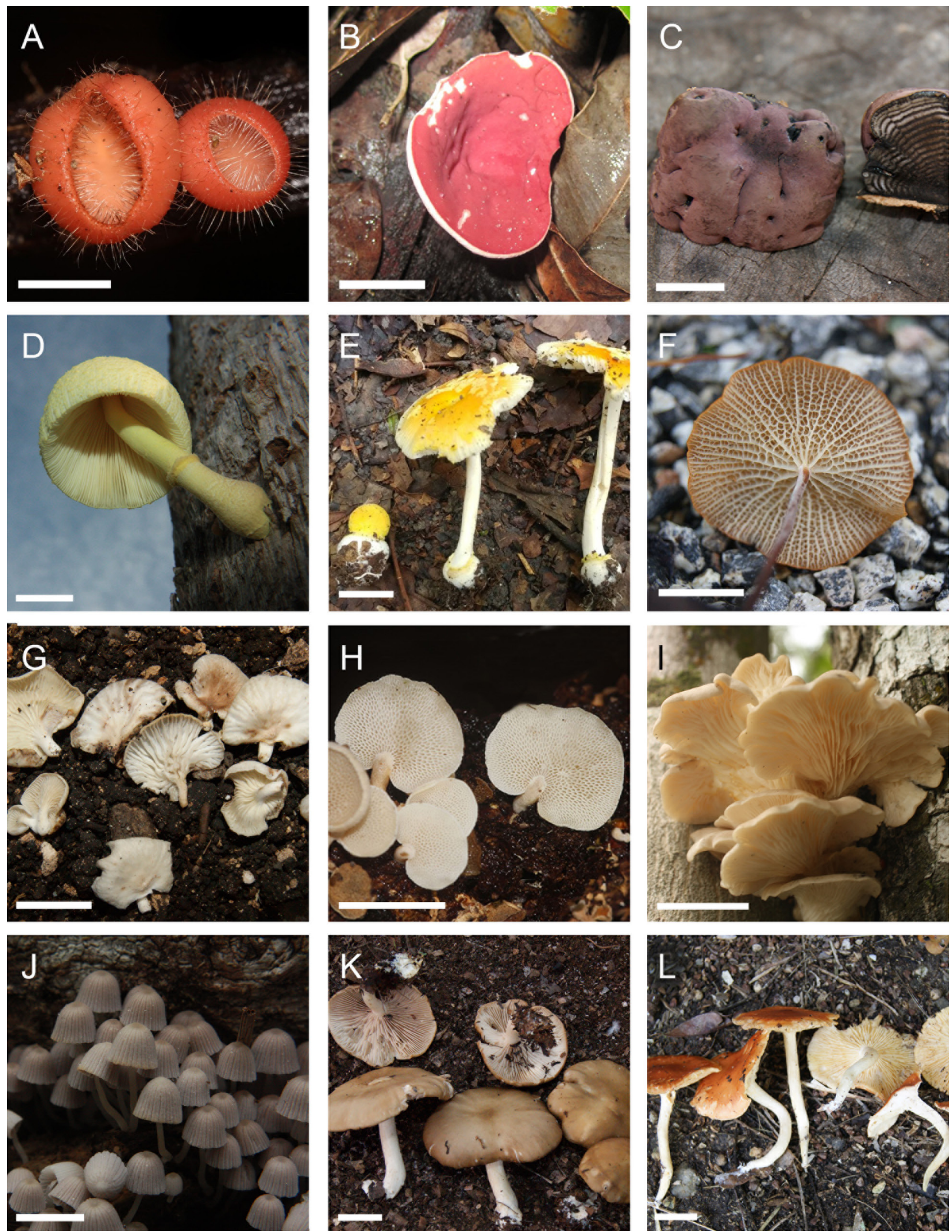

Figure 4. Representative fungi from Quintana Roo. A. Cookeina tricholoma B. Phillipsia domingensis. C. Daldinia eschscholtzii. D. Leucocoprinus birnbaumii. E. Amanita silvatica. F. Marasmius cladophyllus. G. Nothopanus eugrammus. H. Panellus pusillus. I. Pleurotus djamor. J. Coprinellus disseminatus. K. Lepista yucatanensis. L. Leucopaxillus gracillimus. Scale bars $=10 \mathrm{~mm}$.

Xylaria juruensis Henn.

Literature: San Martín and Rogers (1989), Medel et al. (1995).

Xylaria laevis Lloyd.
Literature: San Martín et al. (1995), Medel et al. (1999). Xylaria lancea Lloyd.

Literature: San Martín et al. (1995), Medel et al. (1999). Xylaria magniannulata F. San Martín \& J.D. Rogers. 
Literature: San Martín and Rogers (1988), San Martín et al. (1995), Medel et al. (1999).

*Xylaria mesenterica (Möller) M. Stadler, Læssøe \& J. Fourn. Fig. 2C.

Xylaria microceras (Mont.) Berk.

Literature: San Martín et al. (1995).

Xylaria montagnei Hamme \& Guerrero.

Literature: San Martín et al. (1995), Medel et al. (1999).

Xylaria multiplex (Kunze) Fr.

Literature: Guzmán (1983), San Martín and Rogers (1989), San Martín et al. (1995).

Xylaria muscula Lloyd.

Literature: San Martín and Rogers (1989), Medel et al. (1995).

Xylaria musooriensis Dargan.

Literature: San Martín et al. (1995), Medel et al. (1999).

Xylaria olobapha Berk.

Literature: San Martín et al. (1995).

Xylaria oxyacanthae Tul. \& C. Tul.

Literature: San Martín et al. (1995).

Xylaria pallide-ostiolata Henn.

Literature: San Martín and Rogers (1988), San

Martín et al. (1995), Medel et al. (1999).

Xylaria phyllocharis Mont.

Literature: San Martín and Rogers (1989), San

Martín et al. (1995), Guzmán (2003).

Xylaria poitei (Lév.) Fr.

Literature: San Martín (1995).

Xylaria polymorpha (Pers.) Grev.

Literature: San Martín and Rogers (1989), San

Martín et al. (1995), Guzmán (2003).

Xylaria rhizomorpha (Mont.) Mont.

Literature: San Martín et al. (1995).

Xylaria scabriclavula F. San Martín \& J.D. Rogers.

Literature: San Martín and Rogers (1989), San

Martín et al. (1995).

Xylaria scopiformis Mont. ex Berk. \& Broome. Literature: San Martín et al. (1995).

Xylaria scruposa (Fr.) Fr.

Literature: San Martín et al. (1995).

Xylaria squamulosa F. San Martín \& J.D. Rogers.

Literature: San Martín and Rogers (1989), San

Martín et al. (1995).

Xylaria uniapiculata F. San Martín \& J.D. Rogers.

Literature: San Martín et al. (1995).

\section{Basidiomycota}

\section{Incerta caedis}

Cotylidia aurantiaca (Pat.) A.L. Welden. Literature: Pérez-Silva et al. (1992), Guzmán (2003).

Cotylidia diaphana (Cooke) Lentz.

Literature: Pérez-Silva et al. (1992).

Agaricales

Incertae sedis

Panaeolus antillarum (Fr.) Dennis.

Literature: Guzmán (1983), Chay-Casanova and
Medel (2000), Guzmán (2003).

*Panaeolus cyanescens Sacc. Fig. 2D.

Panaeolus papilionaceus (Bull.) Quél.

Literature: Guzmán (1983).

Panaeolus variabilis Overh.

Literature: Guzmán (1983).

Mesophelliopsis pernambucensis Bat. \& A.F. Vital. Literature: Guzmán (2003).

\section{Agaricaceae}

Agaricus benzodorus Heinem. \& Gooss.-Font.

Literature: Guzmán (1983), Chay-Casanova and

Medel (2000).

Agaricus dennisii Heinem.

Literature: Guzmán (1983), Chay-Casanova and

Medel (2000).

Agaricus dulcidulus Schulzer F.H. Møller.

Literature: Guzmán (1983), Chay-Casanova and

Medel (2000).

Agaricus endoxanthus Berk. \& Broome.

Literature: Guzmán (1983), Chay-Casanova and

Medel (2000).

Agaricus singeri Heinem.

Literature: Guzmán (1983), Chay-Casanova and Medel (2000).

Agaricus trinitatensis R.E.D. Baker \& W.T. Dale.

Literature: Guzmán (1983), Chay-Casanova and

Medel (2000).

Agaricus xanthodermus Genev.

Literature: Pérez-Silva et al. (1992), Chay-Casanova and Medel (2000).

Chlorophyllum molybdites (G. Mey.) Massee.

Literature: Guzmán (2003).

Cyathia intermedia (Mont.) V.S. White.

Literature: Chío and Guzmán (1982).

Cyathus colensoi Berk.

Literature: Guzmán (2003), Pompa-Gonzáles et al. (2011).

Cyathus limbatus Tul. \& C. Tul.

Literature: Guzmán (1983).

Cyathus pallidus Berk. \& M.A. Curtis.

Literature: Guzmán (1983).

Lepiota ciqroensis Guzm.-Dáv. \& Guzmán.

Literature: Guzmán-Dávalos and Guzmán (1982).

Lepiota microcystidiata Guzm.-Dáv. \& Guzmán.

Literature: Guzmán-Dávalos and Guzmán (1982).

Lepiota montagnei (Kalchbr.) Sacc.

Literature: Guzmán-Dávalos and Guzmán (1982).

Lepiota mucronata Guzm.-Dáv. \& Guzmán.

Literature: Guzmán-Dávalos and Guzmán (1982),

Chay-Casanova \& Medel (2000).

Lepiota phaeosticta Morgan.

Literature: Guzmán-Dávalos and Guzmán (1982).

Lepiota pseudoroseola Dennis.

Literature: Guzmán-Dávalos and Guzmán (1982).

Lepiota quintanaroensis Guzm.-Dáv. \& Guzmán.

Literature: Guzmán-Dávalos and Guzmán (1982),

Chay-Casanova and Medel (2000). 
Lepiota spiculata Pegler.

Literature: Singer and García (1988).

Lepiota termitomyces Guzm.-Dáv. \& Guzmán.

Literature: Guzmán-Dávalos and Guzmán (1982).

Lepiota vascularis Pegler.

Literature: Guzmán-Dávalos and Guzmán (1982).

Leucoagaricus lilaceus Singer.

Literature: de la Fuente et al. (2018b).

Leucocoprinus badhamii (Berk. \& Broome) Locq.

Literature: Pérez-Silva et al. (1992).

Leucocoprinus birnbaumii (Corda) Singer.

Literature: Chío and Guzmán (1982), Pompa-

Gonzáles et al. (2011). Fig. 4D.

Leucocoprinus cepistipes (Sowerby) Pat.

Literature: Guzmán (1983).

Lycogalopsis solmsii E. Fisch.

Literature: Guzmán (2003).

Lycoperdon subincarnatum Peck.

Literature: Guzmán (1983).

*Rugosospora ochraceobadia (Beeli) Heinem. Fig. 2E.

Tulostoma brumale Pers.

Literature: Pérez-Silva et al. (1992).

\section{Amanitaceae}

*Amanita arenicola O. Ok Mill. \& Lodge. Fig. 2F.

Amanita gemmata (Fr.) Bertill.

Literature: Pérez-Silva et al. (1992).

Amanita silvatica Guzmán.

Literature: Guzmán (1982), Guzmán (2006). Fig. 4E.

Amanita vaginata (Bull.) Lam.

Literature: Pérez-Silva et al. (1992).

Amanita yucatanensis Guzmán.

Literature: Guzmán (1982).

\section{Bolbitaceae}

Conocybe apala (Fr.) Arnolds.

Literature: Pérez-Silva et al. (1992).

\section{Entolomataceae}

Entoloma atropileatum Dennis.

Literature: Guzmán (1983).

Entoloma bakeri Dennis.

Literature: Guzmán (1983).

Entoloma guatopoanum (Dennis) E. Horak.

Literature: Guzmán (1983).

Entoloma howellii (Peck) Dennis. Literature: Guzmán (1983).

Entoloma lisalense (Beeli) Noordel. \& Co-David. Literature: Guzmán (1983).

Entoloma naranjanum Dennis. Literature: Guzmán (1983).

Entoloma obscurum Dennis.

Literature: Guzmán (1983).

Entoloma sericellum (Fr.) P. Kumm.

Literature: Guzmán (1983).

Entoloma stylophorum (Berk. \& Broome) Sacc.

Literature: Guzmán (1983).

Entoloma umbilicatum Dennis.

Literature: Guzmán (1983).
Entoloma underwoodii Dennis.

Literature: Guzmán (1983).

Entoloma venezuelanum (Dennis) E. Horak.

Literature: Guzmán (1983).

Entoloma violaceum Murrill.

Literature: Guzmán (1983).

Fistulinaceae

*Pseudofistulina radicata (Schwein.) Burds. Fig. 2G.

Hygrophoraceae

Hygrocybe earlei Murrill.

Literature: Guzmán (1983).

Hygrocybe firma (Berk.\& Broome) Singer.

Literature: Guzmán (1983).

Hygrocybe hypohaemacta (Corner) Pegler.

Literature: Guzmán (1983).

Hygrophorus buccinulus (Speg.) Dennis.

Literature: Guzmán (1983).

Hymenogastraceae

Deconica coprophila (Bull.) P. Karst.

Literature: Guzmán (1983).

Gymnopilus lepidotus Hesler.

Literature: Guzmán (2003).

Hebeloma vatricosum (Fr.) Murrill.

Literature: Pérez-Silva et al. (1992).

*Psilocybe cubensis (Earle) Singer. Fig. 2H.

Inocybaceae

Crepidotus decipiens Singer.

Literature: Guzmán (1983).

Inocybe tropicalis Guzmán.

Literature: Guzman (1982), Guzmán (2003).

*Inocybe xerophytica Pegler. Fig. 2I.

Neopaxillus echinospermus (Speg.) Singer.

Literature: Guzmán (1983), Chay-Casanova and

Medel (2000).

*Neopaxillus dominicanus Angelini \& Vizzini. Fig. 2J.

\section{Lycoperdaceae}

Apioperdon pyriforme (Schaeff) Vizzini.

Literature: Guzmán (1983).

\section{Lyophyllaceae}

*Calocybe cyanea Singer ex Redhead \& Singer. Fig. $2 \mathrm{~K}$.

\section{Marasmiaceae}

Baeospora pleurotoides (Dennis) Singer. Literature: Guzmán (1983).

Campanella junghuhnii (Mont.) Singer. Literature: Guzmán (1983).

Clitocybula omphaliiformis Pegler. Literature: Guzmán (1983).

Crinipellis actinophora (Berk. \& Broome) Singer. Literature: Guzmán (2003).

*Gerronema bryogeton Singer. Fig. 2L.

Hemimycena longicystis R. Heim.

Literature: Guzmán (1983).

*Hydropus nigrita (Berk. \& M.A. Curtis) Singer.

Fig. 3A. 
Lactocollybia epia (Berk. \& Broome) Pegler.

Literature: Guzmán (1983).

Marasmius aciculiformis Berk. \& M.A. Curtis.

Literature: Guzmán (1982), Chay-Casanova and Medel (2000).

Marasmius berteroi (Lév.) Murrill.

Literature: Guzmán (1983), Chay-Casanova and Medel (2000).

Marasmius cladophyllus Berk.

Literature: Guzmán (2003). Fig. 4F.

Marasmius crinis-equi F. Muell. ex Kalchbr.

Literature: Pompa-Gonzáles et al. (2011).

Marasmius edwallianus Henn.

Literature: Guzmán (1983).

Marasmius ferrugineus Berk.

Literature: Guzmán (1983), Chay-Casanova and Medel (2000).

Marasmius foliicola Singer.

Literature: Guzmán (1983).

Marasmius fulvoferrugineus Gilliam.

Literature: Pérez-Silva et al. (1992), Chay-Casanova and Medel (2000).

Marasmius haematocephalus (Mont.) Fr.

Literature: Guzmán (1983).

Marasmius jalapensis Murrill.

Literature: Guzmán (1983), Guzmán (2003).

Marasmius pulcherripes Peck.

Literature: Pompa-Gonzáles et al. (2011).

Nothopanus eugrammus (Mont.) Singer.

Literature: Guzmán (1983), Guzmán (2003). Fig. 4G.

Trogia buccinalis (Mont.) Pat.

Literature: Guzmán (2003).

Trogia cantharelloides (Mont.) Pat.

Literature: Guzmán (1983), Guzmán (2003).

\section{Mycenaceae}

Favolaschia fendleri Singer.

Literature: Guzmán (2003).

Filoboletus clypeatus (Pat.) Singer.

Literature: Guzmán (1983).

Filoboletus gracilis (Klotzsch ex Berk.) Singer.

Literature: Guzmán (2003).

Mycena albostriata Dennis.

Literature: Guzmán (1983).

Mycena holoporphyra (Berk. \& M.A. Curtis) Singer.

Literature: Guzmán (1983).

Mycena plectophylla (Mont.) Dennis.

Literature: Guzmán (1983).

Mycena pura (Pers.) P. Kumm.

Literature: Guzmán (1983).

Mycena tessellata (Mont.) Dennis.

Literature: Guzmán (1983).

Panellus pusillus (Pers. ex Lév.) Burds. \& O.K. Mill. Literature: Guzmán (1983), Chay-Casanova and Medel (2000), Guzmán (2003). Fig. 4H.

Xeromphalina tenuipes (Schwein.) A.H. Sm.

Literature: Pérez-Silva et al. (1992), Chay-Casanova and Medel (2000).

\section{Niaceae}

Merismodes ochracea (Hoffm.) D.A. Reid.

Literature: Guzmán (1983), Chay-Casanova and Medel (2000).

\section{Omphallotaceae}

Connopus acervatus (Fr.) K.W. Hughes, Mather \& R.H. Petersen.

Literature: Guzmán (1983).

Gymnopus confluens (Pers.) Antonín, Halling \& Noordel.

Literature: Guzmán (1983).

Gymnopus omphalodes (Berk.)Halling \& J.L. Mata.

Literature: Guzmán (1983).

Gymnopus polyphyllus (Peck) Halling.

Literature: Guzmán (1983).

Marasmiellus catephes (Berk.) Singer.

Literature: Guzmán (1983).

Marasmiellus cubensis (Berk. \& M.A. Curtis) Singer. Literature: Guzmán (2003).

Marasmiellus inoderma (Berk.) Singer.

Literature: Guzmán (1983), Chay-Casanova and

Medel (2000).

Marasmiellus roseipallens (Murrill) Singer.

Literature: Guzmán (1983).

Neonothopanus hygrophanus (Mont.) De Kesel \& Degreef.

Literature: Guzmán (1983), Chay-Casanova and Medel (2000).

\section{Pleurotaceae}

Pleurotus djamor (Rumph. ex Fr.) Boedijn.

Literature: Guzmán (2003), Pompa-Gonzáles et al. (2011). Fig. 4I.

Pleurotus ostreatus (Jacq.) P. Kumm.

Literature: Guzmán (1983), Pérez-Silva et al. (1992), Chay-Casanova and Medel (2000).

\section{Pluteaceae}

Pluteus oligocystis Singer. Literature: Guzmán (1983), Rodríguez (2013).

Pluteus podospileus Sacc. \& Cub. Literature. Pérez-Silva et al. (1992), Chay-Casanova and Medel (2000).

Pluteus pulverulentus Murrill. Literature: Guzmán (1983), Rodríguez (2013).

Volvariella bakeri (Murrill) Shaffer. Literature: Guzmán (1993).

Volvariella bombycina (Schaeff.) Singer. Literature: Guzmán (2003).

Volvariella lepiotospora Singer. Literature: Guzmán (1983).

Volvariella pseudovolvacea (Berk. \& Broome) Singer. Literature: Guzmán (2003).

Volvariella volvacea (Bull.) Singer. Literature: Guzmán (2003).

\section{Psathyrellaceae}

Coprinellus radians (Fr.) Vilgalys, Hopple \& Jacq. Johnson. 
Literature: Guzmán (2003).

Coprinellus disseminatus (Pers.) J.E. Lange.

Literature: Tun-Cano (2018). Fig. 4J.

Coprinopsis echinospora (Buller) Redhead, Vilgalys \& Moncalvo.

Literature: Guzmán (1983), Guzmán (2003).

Coprinopsis lagopus (Fr.) Redhead, Vilgalys \&

Moncalvo.

Literature: Pompa-Gonzáles et al. (2011).

Psathyrella albocapitata Dennis.

Literature: Guzmán (1983), Guzmán et al. (1988).

Psathyrella ammophila (Durieu \& Lév.) P.D. Orton.

Literature: Guzmán (1983), Guzmán et al. (1988).

Psathyrella araguana Dennis.

Literature: Guzmán (1983), Guzmán et al. (1988).

Psathyrella candolleana (Fr.) Maire.

Literature: Guzmán (1983), Guzmán et al. (1988).

Psathyrella coprinoceps (Berk. \& M.A. Curtis) Dennis.

Literature: Guzmán (1983), Guzmán et al. (1988).

Psathyrella erinensis Dennis.

Literature: Guzmán (1983), Guzmán et al. (1988).

Psathyrella mundiliformis (Dennis) Dennis.

Literature: Guzmán (1983), Guzmán et al. (1988).

Psathyrella subnuda (P. Karst.) A.H. Sm.

Literature: Guzmán (1983), Guzmán et al. (1988).

\section{Pterulaceae}

Pterula plumosa (Schwein.) Fr.

Literature: Guzmán (2003).

\section{Schizophyllaceae}

Schizophyllum commune Fr.

Literature: Chío and Guzmán (1982), Chay-Casanova and Medel (2000), Pompa-Gonzáles et al. (2011).

Schizophyllum fasciatum Pat.

Literature: Guzmán (1983), Pérez-Silva et al. (1992),

Chay-Casanova and Medel (2000).

Schizophyllum umbrinum Berk.

Literature: Guzmán (1983), Chay-Casanova and

Medel (2000).

\section{Strophariaceae}

Agrocybe praecox (Pers.) Fayod.

Literature: Pérez-Silva et al. (1992).

Agrocybe retigera (Speg.) Singer.

Literature: Guzmán (1983), Guzmán (2003).

\section{Tricholomataceae}

Arrhenia epichysium (Pers.) Redhead, Lutzoni,

Moncalvo \& Vilgalys.

Literature: Guzmán (1983).

Clitocybe jalapensis (Murrill) Singer.

Literature: Guzmán (1983).

Collybia trinitatis Dennis.

Literature: Guzmán (2003).

Collybia zonata (Peck.)Sacc.

Literature: Pérez-Silva et al. (1992).

Lepista yukatanensis Guzmán \& Bon.

Literature: Guzmán (1982), Guzmán (2003). Fig. 4K.

Leucopaxillus gracillimus Singer \& A.H. Sm.
Literature: Guzmán (2003). Fig. 4L.

*Macrocybe titans (H.E. Bigelow \& Kimbr.) Pegler, Lodge \& Nakasone. Fig. 3B.

Melanoleuca yucatanensis Pegler.

Literature: Guzmán (1983), Chay-Casanova and

Medel (2000), Pompa-Gonzáles et al. (2011),

Sánchez-García et al. (2013).

Tricholomopsis tropica Dennis.

Literature: Guzmán (1983).

\section{Auriculariales}

Auriculariaceae

Auricularia cornea Ehrenb.

Literature: Guzmán (2003).

Auricularia delicata (Mont. ex Fr.) Henn.

Literature: Guzmán (2003).

Auricularia fuscosuccinea (Mont.) Henn.

Literature: Guzmán (2003); Pompa-Gonzáles et al.

(2011). Fig. 5A.

Auricularia mesenterica (Dicks.) Pers.

Literature: Guzmán (2003), Pompa-Gonzáles et al. (2011).

Auricularia nigricans (Sw.) Birkebak, Looney \&

Sánchez-García.

Literature: Guzmán (1983), Pompa-Gonzáles et al. (2011).

Boletales

Boletaceae

Boletellus cubensis (Berk. \& M.A. Curtis) Singer. Literature: Singer et al. (1993), de la Fuente et al. (2018a).

*Mucilopilus mexicanus (Guzmán) Wolfe. Fig. 3C.

Octaviania ciqroensis Guzmán.

Literature: Guzmán (1982), de la Fuente et al. (2018a).

Pulveroboletus aberrans Heinem. \& Gooss.-Font.

Literature: Guzmán (1983).

Xerocomus coccolobae Pegler.

Literature: de la Fuente et al. (2018a).

Xerocomus caeruleonigrescens Pegler.

Literature: García-Jiménez (1999), García-Jiménez

and Garza-Ocañas (2001), de la Fuente et al. (2018a).

Xerocomus cuneipes Pegler.

Literature: García-Jiménez (1999), García-Jiménez

and Garza-Ocañas (2001), de la Fuente et al. (2018a).

Boletinellaceae

Phlebopus brasiliensis Singer.

Literature: de la Fuente et al. (2018a).

Phlebopus colossus (R. Heim) Singer.

Literature: Guzmán (1983), Guzmán (2003), de la

Fuente et al. (2018a).

\section{Coniophoraceae}

*Gyrodontium sacchari (Spreng.) Hjortstam. Fig. 3D.

\section{Sclerodermataceae}

Scleroderma albidum Pat. \& Trab.

Literature: Guzmán et al. (2014), de la Fuente et al. (2018a). 

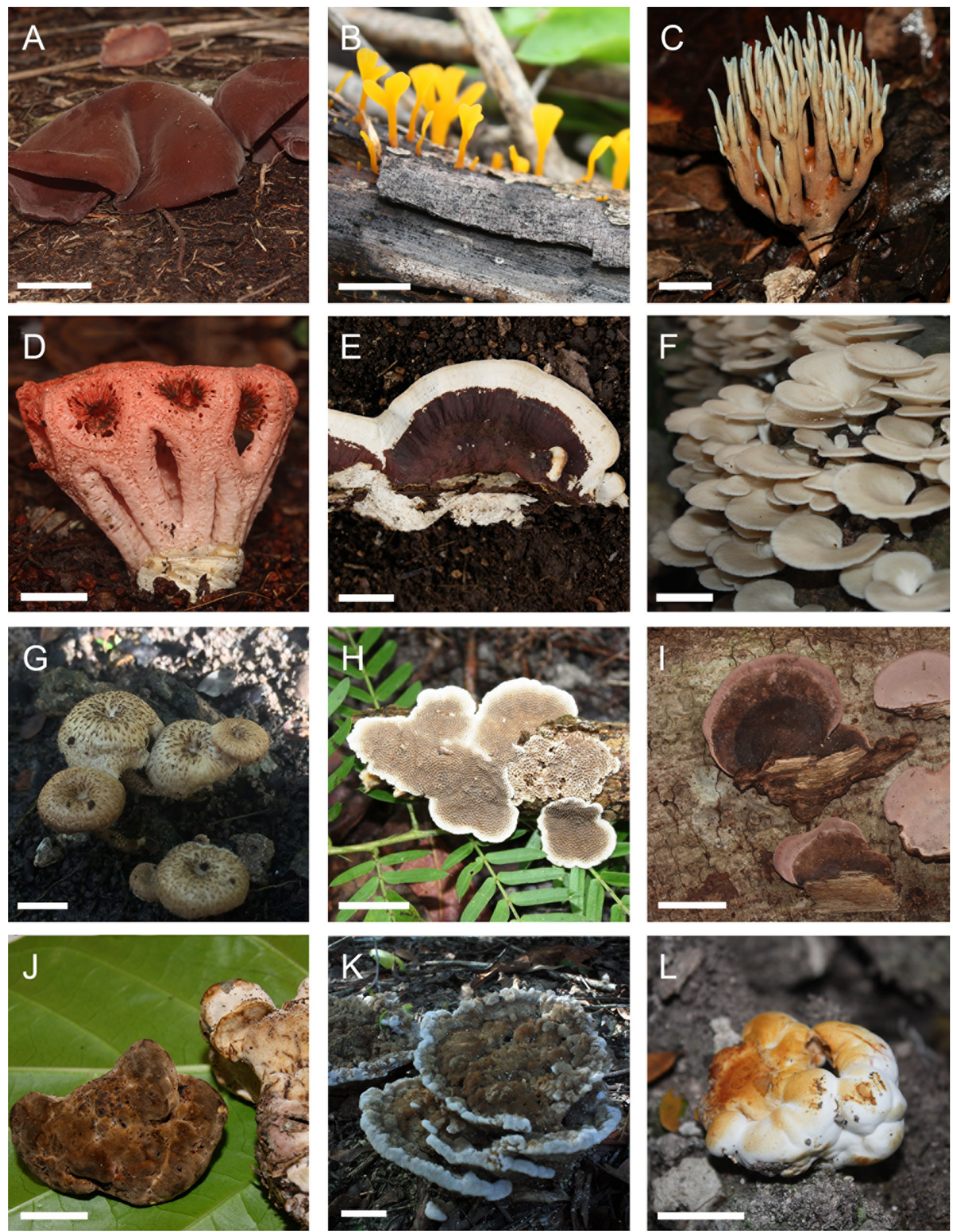

Figure 5. Representative fungi from Quintana Roo. A. Auricularia fuscosuccinea. B. Dacryopinax spathularia. C. Phaeoclavulina cyanocephala. D. Clathrus crispus. E. Earliella scabrosa. F. Favolus tenuiculus. G. Lentinus tigrinus. H. Trametes villosa. I. Rhodofomitopsis feei. J. Amylosporus campbellii. K. Tremelloscypha gelatinosa. L. Redeckera fulva. Scale bars $=10 \mathrm{~mm}$.

Scleroderma areolatum Ehrenb.

Literature: Pérez-Silva et al. (1992), Guzmán et al.

(2014), de la Fuente et al. (2018a).

Scleroderma bermudense Coker.
Literature.Guzmán (1983), Guzmán et al. (2014), de la Fuente et al. (2018a).

Scleroderma nitidum Berk.

Literature: de la Fuente et al. (2018a). 
Scleroderma sinnamariense Mont.

Literature: Chay-Casanova and Medel (2000), PompaGonzáles et al. (2011), de la Fuente et al. (2018a).

\section{Paxillaceae}

Gyrodon intermedius (Pat.) Singer.

Literature: Chío and Guzmán (1982), de la Fuente et al. (2018a).

\section{Suillaceae}

Suillus decipiens (Peck) Kuntze.

Literature: de la Fuente et al. (2018a).

\section{Cantharellales}

\section{Cantharellaceae}

* Cantharellus coccolobae Buyck, P.-A. Moreau \& Courtec. Fig. 3E.

\section{Dacrymycetales}

\section{Dacrymycetaceae}

Calocera cornea (Batsch) Fr.

Literature: Guzmán (2003), Pompa-Gonzáles et al. (2011).

Calocera macrospora Brasf.

Literature: Guzmán (2003).

Cerinomyces lagerheimii (Pat.) McNabb.

Literature: Sierra and Cifuentes (2008).

Dacryopinax elegans (Berk. \& M.A. Curtis) G.W. Martin. Literature: Pérez-Silva et al. (1992), Guzmán (2003). Fig.5B.

Dacryopinax spathularia (Schwein) G. W. Martin. Literature: Pérez-Silva et al. (1992), Guzmán (2003), Pompa-Gonzáles et al. (2011).

Ditiola radicata (Alb. \& Schwein.) Fr. Literature: Guzmán (1983).

\section{Geastrales}

\section{Geastraceae}

Geastrum campestre Morgan. Literature: Guzmán (2003).

Geastrum coronatum Pers.

Literature: Pérez-Silva et al. (1999).

Geastrum fimbriatum Fr.

Literature: Guzmán (2003).

Geastrum fuscogleba (Zeller) Jeppson \& E. Larss. Literature: Pérez-Silva et al. (1993).

Geastrum mirabile Mont.

Literature: Pérez-Silva et al. (1993).

Geastrum pectinatum Pers.

Literature: Guzmán (1983).

Geastrum saccatum Fr.

Literature: Pérez-Silva et al. (1999), Guzmán (2003).

Geastrum schweinitzii (Berk. \& M.A. Curtis) Zeller. Literature: Guzmán (1983), Guzmán (2003).

Geastrum subiculosum Cooke \& Massee.

Literature: Guzmán (1983).

Geastrum triplex Jungh.

Literature: Guzmán (1983).
Myriostoma coliforme (Dicks.) Corda.

Literature: Guzmán (1983), Chay-Casanova and

Medel (2000), Guzmán (2003).

Gloeophyllales

Gloeophyllaceae

Gloeophyllum striatum (Fr.) Murrill.

Literature: Guzmán (1983), Bandala et al. (1993),

Pompa-Gonzáles et al. (2011).

\section{Gomphales}

Gomphaceae

Gomphus brunneus (Heinem.) Corner. Literature: Guzmán (1983).

Phaeoclavulina articulotea (R. H. Petersen) Giachini. Literature: Gonzáles-Ávila et al. (2013).

Phaeoclavulina cyanocephala (Berk. \& M.A. Curtis) Giachini.

Literature: Guzmán (1983), Chay-Casanova and Medel (2000), Guzmán (2003), Gonzáles-Ávila et al. (2013). Fig. 5C.

Phaeoclavulina guyanensis (Pat.) Giachini.

Literature: Guzmán (1983).

Phaeoclavulina zippeli (Lév) Overeem. Literature: Gonzáles-Ávila et al. (2013).

Terenodon serenus Maas Geest. Literature: Guzmán (1983).

\section{Lentariaceae}

Lentaria surculus (Berk.) Corner. Literature: Guzmán (2003).

\section{Hymenochaetales}

Incertae cedis

Trichaptum biforme (Fr.) Ryvarden. Literature: Guzmán (1983), Bandala et al. (1993), Guzmán (2003).

Trichaptum byssogenum (Jungh.) Ryvarden. Literature: Bandala et al. (1993).

Trichaptum fumosoavellaneum (Romell) Rajchenb. \& Bianchin.

Literature: Bandala et al. (1993).

Trichaptum perrottetii (Lév.) Ryvarden. Literature: Pérez-Silva et al. (1992), Bandala et al. (1993), Guzmán (2003).

Trichaptum sector (Ehrenb.) Kreisel. Literature: Pérez-Silva et al. (1992), Chay-Casanova and Medel (2000), Pompa-Gonzáles et al. (2011).

Trichaptum trichomallum (Berk. \& Mont.) Murrill. Literature: Chío and Guzmán (1982).

\section{Hymenochaetaceae}

Fomitiporia robusta (P. Karst.) Fiasson \& Niemelä. Literature: Guzmán (1983).

Fulvifomes rhytiphloeus (Mont.) Camp.-Sant. \& Robledo. Literature: Ryvarden and Guzmán (1993), Bandala et al. (1993).

Fuscoporia contigua (Pers.) G. Cunn. Literature: Ryvarden and Guzmán (1993), Raymundo et al. (2013).

Hymenochaete luteobadia (Fr.) Höhn. \& Litsch. Literature: Tapia et al. (2016). 
Hymenochaete rheicolor (Mont.) Lév.

Literature: Pérez-Silva et al. (1992).

Inocutis porrecta (Murrill) Baltazar.

Literature: Pompa-Gonzáles et al. (2011).

Inonotus luteoumbrinus (Romell) Ryvarden.

Literature: Ryvarden and Guzmán (1993), Bandala

et al. (1993), Guzmán (2003), Pompa-Gonzáles et al. (2011).

Inonotus tabacinus (Mont.) G. Cunn.

Literature: Bandala et al. (1993).

Phellinus crocatus (Fr.) Ryvarden.

Literature: Chío and Guzmán (1982), Guzmán (1983).

Phellinus fastuosus (Lév.) S. Ahmad. Literature: Guzmán (1983), Chay-Casanova and Medel (2000), Pompa-Gonzáles et al. (2011).

Phellinus ferrugineovelutinus (Henn.) Ryvarden. Literature: Ryvarden and Guzmán (1993), ChayCasanova and Medel (2000).

Phellinus gilvus (Schwein.) Pat.

Literature: Guzmán (1983), Pérez-Silva et al. (1992),

Bandala et al. (1993), Guzmán (2003), Pompa-

Gonzáles et al. (2011), Raymundo et al. (2013).

Phellinus grenadensis (Murrill) Ryvarden.

Literature: Ryvarden and Guzmán (1993).

Phellinus nilgheriensis (Mont.) G. Cunn.

Literature: Bandala et al. (1993), Pompa-Gonzáles et al. (2011).

Phellinus rimosus (Berk.) Pilát. Literature: Pérez-Silva et al. (1992), Chay-Casanova and Medel (2000), Guzmán (2003), Pompa-Gonzáles et al. (2011).

Phellinus robinie (Murrill) A. Ames.

Literature: Bandala et al. (1993).

Phellinus roseocinereus (Murrill) D.A. Reid.

Literature: Guzmán (1983), Bandala et al. (1993),

Chay-Casanova and Medel (2000).

Phellinus swieteniae (Murrill) S. Herrera \& Bondartseva. Literature: Chío and Guzmán (1982), Bandala et al. (1993).

Phylloporia chrysites (Berk.) Ryvarden. Literature: Guzmán (2003), Pompa-Gonzáles et al. (2011).

Phylloporia pectinata (Klotzsch) Ryvarden. Literature: Guzman (1983).

Phylloporia spathulata (Hook.) Ryvarden. Literature: Guzmán (1983), Pérez-Silva et al. (1992), Ryvarden and Guzmán (1993), Chay-Casanova and Medel (2000), Guzmán (2003).

Phylloporia verae-crucis (Berk. ex Sacc.) Ryvarden. Literature: Ryvarden and Guzmán (1993), Bandala et al. (1993), Guzmán (2003), Guzmán (2004).

Sanghuangporus weirianus (Bres.)L.W. Zhou \& Y.C. Dai. Literature: Bandala et al. (1993).

Tropicoporus linteus (Berk. \& M.A. Curtis) L.W. Zhou \& Y.C. Dai.

Literature: Guzmán (1983), Bandala et al. (1993),
Chay-Casanova and Medel (2000), Guzmán (2003),

Pompa-Gonzáles et al. (2011), Valenzuela et al. (2013).

\section{Repetobasidiaceae}

Sidera lenis (P. Karst.) Miettinen.

Literature: Ryvarden and Guzmán (1993).

\section{Phallales}

Phallaceae

Clathrus crispus Turpin.

Literature: Guzmán (2003), Pompa-González et al. (2011).

Clathrus ruber P. Micheli ex Pers.

Literature: Pompa-Gonzáles et al. (2011).

*Laternea dringii A. López, D. Martínez \& J. García. Fig. 3F.

Phallus indusiatus Schltdl. Literatura: Guzmán et al. (1990).

\section{Polyporales}

Ganodermataceae

Amauroderma camerarium (Berk.) J.S. Furtado. Literature: Bandala et al. (1993), Ryvarden and Guzmán (1993), Guzmán (2003).

Ganoderma australe (Fr.) Pat. Literature: Guzmán (2003).

Ganoderma colossus (Fr.) C.F. Baker. Literature: Guzmán (1983), Bandala et al. (1993), Guzmán (2003), Torres-Torres et al. (2015).

Ganoderma curtisii (Berk.) Murrill.

Literature: Guzmán (1983), Bandala et al. (1993).

Ganoderma fornicatum (Fr.) Pat.

Literature: Guzmán (1983), Bandala et al. (1993).

Ganoderma lobatum (Cooke) G.F. Atk

Literature: Pérez-Silva et al. (1993).

Ganoderma lucidum (Curtis) P. Karst. Literature: Pérez-Silva et al. (1992), Bandala et al. (1993), Guzmán (2003).

Ganoderma resinaceum Boud. Literature: Bandala et al. (1993).

Ganoderma sessile Murrill. Literature: Guzmán (1983).

Ganoderma weberianum (Bres. \& Henn. ex Sacc.) Steyaert.

Literature: Guzmán (1983).

Humphreya coffeata (Berk.) Steyaert. Literature: Ryvarden and Guzmán (1993), Guzmán (2003), Pompa-Gonzáles et al. (2011).

\section{Phanerochaetaceae}

Antrodiella versicutis (Berk. \& M.A. Curtis) Gilb. \& Ryvarden.

Literature: Bandala et al. (1993), Ryvarden and Guzmán (1993), Guzmán (2003), Pompa-Gonzáles et al. (2011).

\section{Meripilaceae}

Rigidoporus microporus (Sw.) Overeem. Literature: Guzmán (1983), Bandala et al. (1993). 
Rigidoporus vinctus (Berk.) Ryvarden.

Literature: Ryvarden and Guzmán (1993), Bandala et al. (1993), Guzmán (2003).

\section{Meruliaceae}

Bjerkandera adusta (Willd.) P. Karst.

Literature: Bandala et al. (1993), Chío and Guzmán (1982).

Cymatoderma caperatum (Berk. \& Mont.) D.A. Reid. Literature: Guzmán (2003).

Flavodon flavus (Klotzsch) Ryvarden. Literature: Ryvarden and Guzmán (1993), Guzmán (2003), Pompa-Gonzáles et al. (2011), Tapia et al. (2016).

Junghuhnia nitida (Pers.) Ryvarden. Literature: Ryvarden and Guzmán (1993), Bandala et al. (1993), Guzmán (2003), Tapia et al. (2016).

\section{Polyporaceae}

Cerioporus varius (Pers.) Zmitr. \& Kovalenko. Literature: Bandala et al. (1993), Ryvarden and Guzmán (1993).

Coriolopsis brunneoleuca (Berk.) Ryvarden. Literature: Pérez-Silva et al. (1992), Pompa-Gonzáles et al. (2011).

Coriolopsis byrsina (Mont.) Ryvarden. Literature: Bandala et al. (1993), Pompa-Gonzáles et al. (2011).

Coriolopsis occidentalis (Klotzsch) Murrill. Literature: Chío and Guzmán (1982).

Earliella scabrosa (Pers.) Gilb. \& Ryvarden. Literature: Chío and Guzmán (1982), Guzmán (1983), Pérez-Silva et al. (1992), Guzmán (2003), Pompa-Gonzáles et al. (2011). Fig. 5E.

Favolus grammocephalus (Berk.) Imazeki. Literature: Bandala et al. (1993), Pompa-Gonzáles et al. (2011).

Favolus tenuiculus P. Beauv.

Literature: Chío and Guzmán (1982), Guzmán (1983), Chay-Casanova and Medel (2000), Guzmán (2003), Pompa-Gonzáles et al. (2011). Fig. 5F.

Fomes endotheius (Berk.) Sacc. Literature: Chío and Guzmán (1982), Bandala et al. (1993).

Fomes extensus (Lév.) Cooke. Literature: Guzmán (1983), Bandala et al. (1993).

Fomes fasciatus (Sw.) Cooke. Literature: Guzmán (1983).

Fomes meliae (Underw.) Murrill. Literature: Ryvarden and Guzmán (1993), Bandala et al. (1993).

Funalia caperata (Berk.) Zmitr. \& Malysheva. Literature: Chío and Guzmán (1982), Pérez-Silva et al. (1992), Ryvarden and Guzmán (1993), Guzmán (2003).

Funalia floccosa (Jungh.) Zmitr. \& Malysheva. Literature: Ryvarden and Guzmán (1993), Bandala et al. (1993).
Grammothele fuligo (Berk. \& Broome) Ryvarden. Literature: Ryvarden and Guzmán (1993), Guzmán (2003).

Lentinus arcularius (Batsch) Zmitr.

Literature: Pompa-Gonzáles et al. (2011).

Lentinus badius (Berk.) Berk.

Literature: Chío \& Guzmán (1982), Pompa-Gonzáles et al. (2011).

Lentinus berteroi (Fr.) Fr. Literature: Pompa-Gonzáles et al. (2011).

Lentinus crinitus (L.) Fr. Literature: Chío and Guzmán (1982), Guzmán (2003), Pompa-Gonzáles et al. (2011).

Lentinus scleropus (Pers.) Fr. Literature: Pérez-Silva et al. (1992).

Lentinus tigrinus (Bull.) Fr. Literature: Pérez-Silva et al. (1992). Fig. 5G.

Lentinus tricholoma (Mont.) Zmitr. Literature: Chío and Guzmán (1982), Bandala et al. (1993), Chay-Casanova and Medel (2000), Guzmán (2003), Pompa-Gonzáles et al. (2011).

Lentinus velutinus Fr. Literature: Guzmán (2003), Pompa-Gonzáles et al. (2011).

Microporellus obovatus (Jungh.) Ryvarden. Literature: Ryvarden and Guzmán (1993), Bandala et al. (1993), Chay-Casanova and Medel (2000).

Panus rudis Fr. Literature: Chío and Guzmán (1982).

Perenniporia ohiensis (Berk.) Ryvarden. Literature: Ryvarden and Guzmán (1993), Bandala et al. (1993), Chay-Casanova and Medel (2000).

Polyporus albiceps Peck. Literature: Guzmán (1983).

Polyporus guianensis Mont. Literature: Guzmán (1983), Bandala et al. (1993), Guzmán (2003), Pompa et al. (2011).

Polyporus leprieuri Mont. Literature: Guzmán (1983), Bandala et al. (1993).

Pycnoporus sanguineus (L.) Murrill. Literature: Guzmán (1983), Bandala et al. (1993), Chay-Casanova and Medel (2000), Pompa-Gonzáles et al. (2011).

Trametes elegans (Spreng.) Fr. Literature: Guzmán (1983), Bandala et al. (1993), Chay-Casanova and Medel (2000), Pompa-Gonzáles et al. (2011).

Trametes hirsuta (Wulfen) Lloyd. Literature: Guzmán (1983).

Trametes hirta (P. Beauv.)Zmitr., Wasser \& Ezhov. Literature: Chío and Guzmán (1982), Bandala et al. (1993).

Trametes hydnoides (Sw.) M. Fidalgo. Literature: Guzmán (1983), Bandala et al. (1993), Guzmán (2003), Pompa-Gonzáles et al. (2011).

Trametes maxima (Mont.) A. David \& Rajchenb. Literature: Chío and Guzmán (1982), Pérez-Silva et 
al (1992), Bandala et al. (1993), Chay-Casanova and Medel (2000), Pompa-Gonzáles et al. (2011).

Trametes membranacea (Sw.) Kreisel.

Literature: Guzmán (1983), Pérez-Silva et al. (1992),

Bandala et al. (1993), Chay-Casanova and Medel (2000).

Trametes modesta (Kunze ex Fr.) Ryvarden.

Literature: Chío and Guzmán (1982).

Trametes pavonia (Berk.) Fr.

Literature: Pérez-Silva et al. (1992), Chay-Casanova and Medel (2000), Pompa-Gonzáles et al. (2011).

Trametes polyzona (Pers.) Justo.

Literature: Bandala et al. (1993), Chay-Casanova and Medel (2000), Pompa-Gonzáles et al. (2011).

Trametes tenuis (Fr.) Fr.

Literature: Bandala et al. (1993), Guzmán (2003).

Trametes variegata (Berk.) Zmitr., Wasser \& Ezhov.

Literature: Guzmán (1983), Bandala et al. (1993),

Chay-Casanova and Medel (2000), Guzmán (2003),

Pompa-Gonzáles et al. (2011).

Trametes villosa (Sw.) Kreise.

Literature: Guzmán (1983), Pérez-Silva et al. (1992),

Bandala et al. (1993), Chay-Casanova and Medel

(2000), Pompa-Gonzáes et al. (2011). Fig. 5H.

\section{Fomitopsidaceae}

Daedalea microsticta Cooke.

Literature: Ryvarden and Guzmán (1993), Bandala et al. (1993), Guzmán (2003).

Phaeodaedalea incerta (Curr.) Ţura, Zmitr., Wasser \& Spirin.

Literature: Guzmán (1983), Chay-Casanova and Medel (2000), Bandala et al. (1993), Guzmán (2003),

Pompa-Gonzáles et al. (2011).

Postia caesia (Schrad.) P. Karst.

Literature: Guzmán (1983), Bandala et al. (1993).

Rhodofomitopsis feei (Fr.) B.K. Cui, M.L. Han \& Y.C.

Dai.

Literature: Chío and Guzmán (1982), Guzmán

(1983), Bandala et al. (1993), Chay-Casanova and

Medel (2000), Guzmán (2003), Pompa-Gonzáles et al. (2011). Fig. 5I.

\section{Phanerochaetaceae}

Ceriporiopsis mucida (Pers.) Gilb. \& Ryvarden. Literature: Tapia et al. (2016).

\section{Russulales}

Bondarzewiaceae

Amylosporus campbellii (Berk.) Ryvarden. Literature: Ryvarden and Guzmán (1993), PompaGonzáles et al. (2011). Fig. 5J.

Stecchericium seriatum (Lloyd) Maas Geest. Literature: Guzmán (1983), Chay-Casanova and Medel (2000), Tapia et al. (2016).

\section{Russulaceae}

*Lactifluus nebulosus (Pegler) De Crop. Fig. 3G.

*Russula cremeolilacina Pegler. Fig. $3 \mathrm{H}$.

\section{Stephanosporaceae}

Mayamontana coccolobae Castellano, Trappe \& Lodge. Literature: de la Fuente et al. 2018.

Stephanospora mayana de la Fuente, García-Jiménez, Guevara-Guerrero \& Oros-Ortega.

Literature: de la Fuente et al. 2019.

\section{Stereaceae}

Xylobolus subpileatus (Berk. \& M.A. Curtis) Boidin. Literature: Pérez-Silva et al. (1992).

\section{Sebacinales}

Sebacinaceae

Sebacina confusa R. Kirschner \& Oberw.

Literature: Guzmán (1983), Guzmán (2003).

Tremelloscypha gelatinosa (Murrill) Oberw. \& K.

Wells.

Literature: Guzmán (2003). Fig. 5K.

Thelephorales

Thelephoraceae

Thelephora pseudoterrestris Corner.

Literature: Guzmán (2003).

Trechisporales

Hydnodontaceae

Hydnodon thelephorus (Lév.) Banker. Literature: Guzmán (2003).

Tremellales

Tremellaceae

*Tremella fuciformis Berk. Fig. 3I.

Tremella mesenterica Retz.

Literature: Guzmán (1983), Chay and Medel (2000).

Tremella rubromaculata Lowy.

Literature: Guzmán (1983), Guzmán (2003), Pompa-

Gonzáles et al. (2011).

Tremella wrightii Berk. \& M.A. Curtis.

Literature: Guzmán (2003).

\section{Glomeromycota}

Diversisporales

Diversisporaceae

Redeckera fulvum (Berk. \& Broome) C. Walker \& A. Schüßler.

Literature: Guzmán (2003), Pompa-Gonzáles et al. (2011). Fig. 5L.

New records of macrofungi for Quintana Roo, Mexico

Ophiocordyceps melolonthae (Tul. \& C. Tul.) G.H. Sung, J.M. Sung, Hywel-Jones \& Spatafora

Material studied. MEXICO - Quintana Roo • J.I. de la Fuente 73 (ITCV); Hermenegildo Galeana; $18^{\circ} 10^{\prime} \mathrm{N}$, $089^{\circ} 14^{\prime} \mathrm{W}$; alt. $229 \mathrm{~m}$; 12 Oct. 2014.

Habit and habitat. Scattered on larvae of Coleoptera in evergreen tropical forest.

Identification. This species is characterized by scattered, clavate, yellowish ascomata, 1-5 cm long, filiform ascospores reaching up to $200 \mu \mathrm{m}$, fragmenting into 
small segments, and the parasitic habit on coleopteran caterpillars. Our material agrees with the descriptions provided by Webber and Smith (1985) and Pérez-Silva et al. (1977).

\section{Geodina guanacastensis Denison}

Material studied. MEXICO - Quintana Roo - J.I. de la Fuente 362 (ITCV); Chetumal; $18^{\circ} 31^{\prime} \mathrm{N}, 088^{\circ} 18^{\prime} \mathrm{W}$; alt. 8 m; 18 Oct. 2019.

Habit and habitat. Caespitose or solitary on humus in lowland forest.

Identification. This species can easily be recognized by orange cup-shaped ascomata covered by brownish hairs and a pseudostipe. Microscopically, this species can be recognized by reticulate spores of $24-35 \times 12-17 \mu \mathrm{m}$. Our material agrees with the description provided by Ortega-López et al. (2019). Our material is morphologicaly similar to G. salmonicolor Angelini \& Medardi from the Dominican Republic (Angelini et al. 2018), which was considered as a synonym of G. guanacastensis by Pfister et al. (2020).

\section{Xylaria mesenterica (Möller) M. Stadler, Læssøe \& J. Fourn.}

Material studied. MEXICO - Quintana Roo • J.I. de la Fuente 68 (ITCV); Jaguactal; $18^{\circ} 14^{\prime} \mathrm{N}, 088^{\circ} 57^{\prime} \mathrm{W}$; alt. 132 m; 12 Oct. 2014.

Habit and habitat. Caespitose on fallen logs in evergreen tropical forest.

Identification. This species can be recognized by the globose and gelatinose stroma with greyish hues and the dark ellipsoid ascospores of 11-14 × 5-6 $\mu \mathrm{m}$. Our material agrees with the description provided by San Martín and Lavín (1997) described for Entonaema pallidum G.W. Martin. This species differs from similar species, such as E. globosum R. Heim. and E. liquescens Möller, by the greyish colour of the stromata. Entonaema pallidum was transferred to Xylaria mesenterica based on molecular and morphological evidence (Stadler et al. 2008).

\section{Panaeolus cyanescens Sacc.}

Material studied. MEXICO - Quintana Roo • J.I. de la Fuente and Aragón 310 (ITCV); Chetumal; 18³1'N, $088^{\circ} 18^{\prime} \mathrm{W}$; alt. 8 m; 27 Sep. 2017.

Habitat and habit. Scattered on humus on secondary vegetation.

Identification. This species can be recognized by the whitish pileus, greyish lamellae, bluish reaction when cut, and subglobose, dark brown basidiospores of 9-12 $\times 6-9 \mu \mathrm{m}$. The material agrees with the description provided by Pegler (1983). It differs from P. antillarum (Fr.) Dennis by the bluish reaction (negative in P. antillarum) the size of the basidioma and the lack of metuloid cystidia (absent in P. antillarum).

\section{Rugosospora ochraceobadia (Beeli) Heinem.}

Material studied. MEXICO - Quintana Roo • J.I. de la Fuente 159 (ITCV); Oxtankáh; $18^{\circ} 36^{\prime} \mathrm{N}, 088^{\circ} 13^{\prime} \mathrm{W}$; alt. 14 m; 17 Oct. 2016.

Habit and habitat. Scattered on humus in evergreen tropical forest.

Identification. This species can be recognized by a white pileus with orange scales, the free white lamellulae, the withish stipe, and the rough spores of 9-13 $\times 6-7$ $\mu \mathrm{m}$. Our material agrees with the descriptions provided by from Ferreira and Cortez (2011) and Bautista-Hernández and Aguirre-Acosta (2004). Rugosospora ochraceobadia differs from $R$. pseudorubiginosa (Cifuentes \& Guzmán) Guzmán \& Bandala by the reticulate spores.

\section{Amanita arenicola O. K. Miller \& Lodge}

Material studied. MEXICO - Quintana Roo - J.I. de la Fuente 427 (ITCV); Chetumal; $18^{\circ} 31^{\prime} \mathrm{N}, 088^{\circ} 18^{\prime} \mathrm{W}$; alt. 8 m; 14 Oct. 2018.

Habit and habitat. Scattered under Coccoloba uvifera in coastal vegetation.

Identification. This species can be recognized by the grey pileus with white patches that are the remains of the volva, white and free lamellulae, a whitish pileus sometimes with conspicuous white scales on the base, the small saccate volva, which sometimes remains underground, and the ellipsoid basidiospores of 8-10 $\times 6-8$ $\mu \mathrm{m}$. Our specimen from Quintana Roo agrees with the description provided by Miller et al. (2000), but it presents slightly shorter spores. Amanita agluttinata (Berk. \& M.A. Curtis) Lloyd is a similar species but differs by the presence of a ring on the stipe (Pegler 1983).

\section{Pseudofistulina radicata (Schwein.) Burds.}

Material studied. MEXICO - Quintana Roo - J.I. de la Fuente 351 (ITCV); Oxtankáh; $18^{\circ} 36^{\prime} \mathrm{N}, 088^{\circ} 13^{\prime} \mathrm{W}$; alt. 14 m; 24 Oct. 2017.

Habit and habitat. Solitary on underground rotten logs in evergreen tropical forest.

Identification. This species can be recognized by the petaloid to reniform pileus, the small whitish rounded pores, and the lateral stipe inserted on the substrate, the subglobose basidiospores of 3-5 $\mu \mathrm{m}$, and the clavate and diverticulate acanthophyses. Our material agrees with the description provided by Guzmán (1987). This species is consumed by the Maya people in Guatemala (Guzmán 1987).

\section{Psilocybe cubensis (Earle) Singer}

Material studied. MEXICO - Quintana Roo - J.I. de la Fuente 173 (ITCV); Blasillo; $18^{\circ} 09^{\prime} \mathrm{N}, 088^{\circ} 57^{\prime} \mathrm{W}$; alt. 132 m; 11 Dec. 2017.

Habit and habitat. Solitary or scattered on cow dung in secondary vegetation. 
Identification. This species is recognized by the mamillate pileus, whitish with yellowish tints and small scales, the free greyish lamellae, the whitish stipe with a dark glutinose ring, the bluish reaction when cut, and the thick-walled, brownish, ellipsoid spores of 10-15 × 6-9 $\mu \mathrm{m}$. Our material agrees with the descriptions provided by Pegler (1983) and Pulido (1983).

\section{Neopaxillus dominicanus Angelini \& Vizzini}

Material studied. MEXICO - Quintana Roo - J.I. de la Fuente 38 (ITCV); San Pedro Peralta; $18^{\circ} 42^{\prime} \mathrm{N}$, $088^{\circ} 49^{\prime} \mathrm{W}$; alt. $175 \mathrm{~m}$; 14 Oct. 2014.

Habit and habitat. Scattered on humus in secondary vegetation.

Identification. This species can be recognized by the pale brown to orange pileus, the whitish decurrent lamellae, and the echinulate rounded spores of $10-13 \mu \mathrm{m}$ in diameter. Our material agrees with the description provided by Vizzini et al. (2011). Neopaxillus echinospermus (Speg.) Singer is a similar species, but it differs from $N$. dominicanus by having an umbilicate pileus.

\section{Calocybe cyanea Singer ex Redhead \& Singer}

Material studied. MEXICO - Quintana Roo - J.I. de la Fuente and Yuridia López 72 (ITCV); Chetumal; $18^{\circ} 31^{\prime} \mathrm{N}, 088^{\circ} 18^{\prime \prime} \mathrm{W}$; alt. $8 \mathrm{~m}$; 05 Oct. 2011.

Habit and habitat. Scattered on humus in secondary vegetation.

Identification. This species can be identified by the purple pileus and the free yellowish lamellae, the greyish stipe, microscopically shows globose spores $3-5 \times$ 2-4 $\mu \mathrm{m}$, hyaline in $\mathrm{KOH}$ without cystidia. Our material agrees with the descriptions provided by GuevaraGuerrero et al. (1985) and Baroni et al. (1999), but our material has more intensely yellow lamellae. Calocybe cyanocephala (Pat.) Pegler is a similar species but with lilac lamellae. Calocybe cyanella Singer ex Redhead \& Singer also has a purple pileus but its lamellae are white (Baroni et al. 1999).

\section{Gerronema bryogeton Singer}

Material studied. MEXICO - Quintana Roo - J.I. de la Fuente 430 (ITCV); road to El Huasteco; $19^{\circ} 18^{\prime} \mathrm{N}$, $088^{\circ} 16^{\prime} \mathrm{W}$; alt. $33 \mathrm{~m}$; 08 Jan. 2019.

Habit and habitat. Scattered on fallen logs in evergreen tropical forest.

Identification. This species can be identified by a small depressed pileus with greyish tones, whitish lamellae, a dark stipe, and ellipsoid spores of $6-9 \times 3-5 \mu \mathrm{m}$. Our material agrees with the description provided by Pegler (1983). Gerronema cyathiforme (Berk. \& M.A. Curtis) is a similar species but differs from G. bryogeton by having a reddish to olivaceous brown stipe (Pegler 1983).

\section{Inocybe xerophytica Pegler}

Material studied. MEXICO - Quintana Roo • J.I. de la
Fuente 317 (ITCV); Chetumal; $18^{\circ} 31^{\prime} \mathrm{N}, 088^{\circ} 18^{\prime} \mathrm{W}$; alt. 8 m; 06 Oct. 2017.

Habit and Habitat. Scattered under Coccoloba uvifera in coastal and secondary vegetation.

Identification. This species can be identified by the flat pileus covered by brown scales, the pale brown lamellae, the pale brown, pruinose stipe without volva, and the subglobose, angulose to verrucose basidiospores of 7-9 $\times 5-7 \mu \mathrm{m}$. Our material agrees with the description provided by Pegler (1983). Inocybe paralanuginosa Pegler is a similar species that differs by having a smooth stipe.

\section{Hydropus nigrita (Berk. \& M.A. Curtis) Singer}

Material studied. MEXICO - Quintana Roo - J.I. de la Fuente 266 (ITCV); Laguna Guerrero; $18^{\circ} 45^{\prime} \mathrm{N}$, 088 $11^{\prime} \mathrm{W}$; alt. 9 m; 11 Jan. 2017.

Habit and habitat. Scattered on fallen logs in lowland forest.

Identification. This species can easily be identified by the olive-greenish pileus with black spots and the cream context that when cut stains black and sometimes exudes a latex-like substance. The material agrees with the description provided by Pegler (1983). The blackening context distinguishes this species from all other tropical Hydropus species.

\section{Macrocybe titans (H.E. Bigelow \& Kimbr.) Pegler, Lodge \& Nakasone}

Material studied. MEXICO - Quintana Roo - J.I. de la Fuente and Quintal Palomo 299 (ITCV); Kohunlich; $18^{\circ} 25^{\prime} \mathrm{N}, 088^{\circ} 47^{\prime} \mathrm{W}$; alt. $120 \mathrm{~m}$; 12 Sep. 2017.

Habit and habitat. Caespitose on humus in evergreen tropical forest.

Identification. This species can be identified by the big size of its basidiomata, the caespitose habit, the crowded white lamellae, and the thick stipe with brown scales. Microscopically it is distinguished by the globose spores, 5.9-8.3 $\times 4.3-5.9 \mu \mathrm{m}$, the ventricose cheilocystidia, the lack of pleurocystidia, and the presence of clamp-connections. Our material agrees with the description provided by Guzmán and Piepenbring (2011) but presents smaller cystidia.

\section{Gyrodontium sacchari (Spreng.) Hjortstam}

Material studied. MEXICO - Quintana Roo • J.I. de la Fuente 298 (ITCV); Chetumal; $18^{\circ} 31^{\prime} \mathrm{N}, 088^{\circ} 18^{\prime} \mathrm{W}$; alt. 8 m; 17 Jan. 2017.

Habit and habitat. Resupinate on dead trees in secondary vegetation.

Identification. This species can be recognized by the resupinate basidiomata with brownish colours, the hymenia composed by truncate spines, which are yellowish when young, becoming olive brown when aged, and the globose, brown spores, 4.0-5.0 × 2.5-3.6 $\mu \mathrm{m}$. Our material agrees with the descriptions provided by from Valenzuela et al. (2014) and Hembrom et al. (2014). 


\section{Mucilopilus mexicanus (Guzmán) Rolfe}

Material studied. MEXICO - Quintana Roo - J.I. de la Fuente 29 (ITCV); Hermenegildo Galeana; $18^{\circ} 10^{\prime} \mathrm{N}$, 089¹4'W; alt. 229 m; 29 Aug. 2014.

Habit and habitat. Solitary or scattered in lowland forest and pine savanna, sometimes associated with Coccoloba or Gymnoponium species.

Identification. This species can be recognized by the greyish pileus, slightly tomentose when young, viscid when aged, the pinkish pores which become grey when aged, the viscid stipe, the fusoid spores $8.1-12.2 \times 4.1-$ $5.9 \mu \mathrm{m}$, and the clavate cystidia. The specimen agrees with the description provided by Guzmán (1974). This species resembles Fistulinella gloeocarpa Pegler in having the basidioma viscid, but it differs from that species by the spore size and the ampullaceous cystidia which has a long slender neck (Pegler 1983).

\section{Cantharellus coccolobae Buyck, P.A. Moreau \& Courtec.}

Material studied. MEXICO - Quintana Roo • J.I. de la Fuente 422 (ITCV); Chetumal; $18^{\circ} 31^{\prime} \mathrm{N}, 088^{\circ} 18^{\prime} \mathrm{W}$; alt. 8 m; 08 Oct. 2018.

Habit and habitat. Scattered under Coccoloba uvifera in coastal vegetation.

Identification. This species can be distinguished by the orange basidiomata, the whitish context which becomes slightly yellowish at the base, slightly peppery flavor, and the ellipsoid basidiospores of 7-11 × 4-6 $\mu \mathrm{m}$. Our material agrees with the description provided by Buyck et al. (2016). Cantharellus cinabarinus (Schwein.) Schwein is a similar species, but it differs by its wider spores and elements of the pileipellis (Pegler 1983; Buyck et al. 2016).

Laternea dringii A. López, D. Martínez \& J. García.

Material studied. MEXICO - Quintana Roo • R.Y. Vela 105 (ITZM); San Pedro Peralta; $18^{\circ} 42^{\prime} \mathrm{N}, 088^{\circ} 49^{\prime} \mathrm{W}$; alt. 175 m; 10 May 2018.

Habit and habitat. Solitary on humus in evergreen tropical forest.

Identification. This species can be distinguished by small basidiomata reaching $2 \mathrm{~cm}$ in length, composed of 3 or 4 yellowish to orange branches, the subtropical distribution, and the small bacillate basidiospores of 4-5 $\times$ $1-1.5 \mu \mathrm{m}$. Our material agrees with the description provided by López et al. (1981). Laternea pusilla Berk. \& M.A. Curtis is a tropical to subtropical species, which differs by having a reddish basidiomata (TrierveilerPereira et al. 2019).

\section{Lactifluus nebulosus (Pegler) De Crop.}

Material studied. MEXICO - Quintana Roo • J.I. de la Fuente 210 (ITCV); Hermenegildo Galeana; $18^{\circ} 10^{\prime} \mathrm{N}$, $089^{\circ} 14^{\prime} \mathrm{W}$; alt. 229 m; 25 jan 2016.
Habit and habitat. Scattered on humus under Gymnopodiumfloribundum and Coccolovadiversifolia in lowland forest.

Identification. This species can be identified by the greyish to cream pileus, slightly wrinkled when aged, the whitish lamellae which stain brown when touched, the peppery white latex, and the ovoid basidiospores 8-11 × 6-9 $\mu \mathrm{m}$ with small verrucae, not reticulated. Our material agrees with the descriptions provided by Pegler and Fiard (1979) and Pegler (1983). Lactifluus caribaeus (Pegler) Verbeken has a similar pileus coloration, but it has a remarkably bad odour (Pegler and Fiard 1979). Lactifluus chiapanensis (Montoya, Bandala \& Guzmán) De Crop also grows under G. floribundum but has smaller spores (8-8.8 $\mu \mathrm{m})$ (Montolla-Bello 1994).

\section{Russula cremeolilacina Pegler}

Material studied. MEXICO - Quintana Roo - J.I. de la Fuente 82 (ITCV); Chetumal; $18^{\circ} 31^{\prime} \mathrm{N}, 088^{\circ} 18^{\prime} \mathrm{W}$; alt. 8 m; 01 Jul. 2014.

Habit and habitat. Scattered under Coccoloba uvifera in coastal vegetation.

Identification. This species can be recognized by the cream pileus that becomes lilac towards the margin, the white, fragile lamellae without lamellulae, the association with Coccoloba species, and the verrucose, subreticulate, subglobose basidiospores of $6-8 \times 5-6.5 \mu \mathrm{m}$. Our material agrees with the description provided by Pegler (1983) but presents paler basidiomata. Russula diversicolor Pegler is a tropical species but it differs in having a yellowish basidioma (Pegler 1983).

\section{Tremella fuciformis Berk.}

Material studied. MEXICO - Quintana Roo - J.I. de la Fuente 308 (ITCV); Bacalar; $18^{\circ} 45^{\prime} \mathrm{N}, 088^{\circ} 20^{\prime} \mathrm{W}$; alt. 19 m; 15 Sep. 2017.

Habit and habitat. Resupinate on dead logs in evergreen tropical forest.

Identification. This species is characterized by gelatinose basidiomata with whitish tones, foliose lobules, a slightly yellowish base, and ovoid to subglobose basidiospores of 5-7 $\times 3.5-5.5 \mu \mathrm{m}$. Our material agrees with the description provided by Weber and Smith (1986). It differs from other Tremella species present in Quintana Roo, like T. rubromaculata Lowy and T. wrightii Berk. \& M.A. Curtis, by the whitish basidioma (Guzmán 2003).

\section{Discussion}

As a result of our fieldwork, which added 21 species for Quintana Roo, 401 species of macrofungi and funguslike organisms are now known for this state. From the literature, 380 species were previously known.

Most species studied here show affinity to fungi of the Neotropical region. They are similar to those previously 
recorded for tropical forests of northern Mexico (García and Valenzuela 2005), the Pacific coast (BautistaHernández and Aguirre-Acosta 2004; Ramírez-López et al. 2012), southern Mexico (Villarruel-Ordáz et al. 2015, López-Guzmán et al. 2017), Central America (Singer et al. 1983; Ortíz-Santana et al. 2007), and the Antilles (Pegler and Fiard 1979; Pegler 1983; Guzmán 1988; Miller et al. 2000; Séne et al. 2012). Some species have wide geographic distributions and occur in Asia and elsewhere in the Americas. For example, Gyrodontium sacchari is found in Asia, South America, and reaches its northernmost distribution in Sonora, Mexico (Hembrom et al. 2014; Robledo et al. 2014; Valenzuela et al. 2014), Cookeina tricholoma and Cookeina speciosa, are pantropical (Denison 1969, 1970; Beug et al. 2013), and Leucoagaricus lilaceus is common in South America (Singer and Digilio 1954; Rother and da Silveira 2009), occurring north to the tropical forests of Tamaulipas (de la Fuente et al. 2018c).

Most species recorded for Quintana Roo are saprobic and very few mycorrhizal species can be found, mostly associated with Polygonaceae and Pinaceae. The most representative species are Amanita arenicola, Cantharellus coccolobae, Inocybe xerophytica, and Russula cremeolilacina, which are associated with Coccoloba uvifera trees in Mexico and the Antilles (Pegler 1983; de la Fuente et al. 2018a); Lactarius nebulosus, associated with Polygonaceae trees in the Antilles and northern Mexico (Pegler and Fiard 1979; García and Valenzuela 2005); Boletellus cubensis, associated with Fabaceae and Polygonaceae (Singer et al. 1993; Ortíz-Santana et al. 2007) and Suillus decipiens, very common in Central America, Cuba, and Florida (Singer 1949; Singer et al. 1983; Kropp 2001; Ortíz-Santana et al. 2007) and also in Quintana Roo (de la Fuente et al. 2018a).

The saprobic species are common in the six vegetation types studied here. Most species develop on dead logs, branches, leaves, or humus and belong to the orders Agaricales, Polyporales, and Xylariales; these orders are the most common in the Mexican tropical forest (Ramírez-López et al 2012). The most representative species are Pycnoporus sanguineus, Schizophyllum commune, Trogia cantharelloides, Trametes villosa, Trametes hydnoides, and numerous species of Geastrum, Hypoxylon, Marasmius, and Xylaria. Parasitic macrofungi are less abundant, but some species of Ganoderma, Phellinus, and Phylloporia grow on Fabaceae trees, mainly on Lysiloma latisiliquum.

The fungal endemism of Quintana Roo has been little discussed (López et al. 2011). However, in this work we present some species that could be endemic to the Yucatan Peninsula. Although the species of Lepiota are very common in tropical forests, some species such as L. ciqroensis, L. microcystidiata, L. mucronata, L. quintanaroensis, and L. termitomyces are only known from Quintana Roo (Guzmán-Dávalos and Guzmán 1982; Chay and Medel 2000). Amanita silvatica has only been reported for Quintana Roo by Guzmán (1983), Guzmán
(2003), and in this work. Mucilopilus mexicanus has been reported once by Guzmán (1974) in Campeche and in this study. Amanita silvatica and Mucilopilus mexicanus are restricted to lowland forests, possibly associated with Coccoloba or Gymnopodium trees. Three trufflelike fungal species show restricted distribution: Mayamontana coccolobae is only known from Belize and southern parts of Campeche and Quintana Roo (Castellano et al. 2007; de la Fuente et al. 2018b). The recently described Stephanospora mayana is reported from Quintana Roo and Campeche growing in lowland forest and pine savanna (de la Fuente et al. 2019). Octaviania ciqroensis is only known from the type collection from northern Quintana Roo (Guzmán 1982). More fieldwork is needed to know the real distribution of these species.

\section{Acknowledgements}

The first author and Ibarra Garibay thank CONACYT for financial support. García-Jiménez and Guevara-Guerrero thank TCN-ITCV-PRODEP for financial support. Garza-Ocañas thanks to UANL for financial support. We kindly thank to Florencia Ramírez-Guillen from Instituto de Ecología (INECOL) for her suggestions and Romeo Sánchez Zavalegui for technical support. We also thank to the reviewers and their editor for their suggesting improvements to the manuscript.

\section{Authors' Contributions}

JIF, JACC, RVH and LEIG collected the specimens, FGO, JGJ, GGG, VMB and JIF identified the species. CYL, GGG, JIF, IOO and JACC wrote the article. LEIG made the figures. All the authors revised the manuscript.

\section{References}

Aguirre-Acosta CE, Ulloa M, Aguilar S, Cifuentes J, Valenzuela R (2014) Biodiversidad de hongos en México. Revista Mexicana de Biodiversidad 85: 76-81. https://doi.org/10.7550/rmb.33649

Angelini C, Medardi G, Alvarado P (2018) Contribution to the study of netropical discomycetes: a new species of the genus Geodina (Geodina salmonicolor sp. nov.) from the Dominican Republic. Mycosphere 9: 169-177. https://doi.org/10.5943/mycosphere/9/2/1

Bandala VM, Guzmán G, Montoya L (1993) The polypores known from Mexico. In: Marmolejo JG, Garza-Ocañas F (Eds) Contribuciones micológicas en homenaje al Biólogo José Castillo Tovar por su labor en pro de la micología mexicana. Reporte especial 13 UANL, Linares, 1-55.

Bandala VM, Montoya L (2015) Gymnopodium floribundum trees (Polygonaceae) harbour a diverse ectomycorrhizal fungal community in the tropical deciduous forest of southeastern Mexico. Research and Reviews: Journal of Botanical Sciences 4: 73-75.

Bautista-Hernández S, Aguirre-Acosta CE (2004) Algunas especies de lepiotáceos de la Estación de Biología Chamela, Jalisco, México. Revista Mexicana de Micología 18: 39-45.

Beug MW, Bessette AE, Bessette AR (2013) Ascomycetes fungi of North America. University of Texas Press, Austin, 488 pp.

Chay-Casanova JA, Medel R (2000) Hongos citados para el jardín botánico y áreas adyacentes. In: Sánchez O, Islebe G (Eds) El jardín botánico Dr. Alfredo Barrera Marín. Fundamentos y estudios particulares. Conabio-Ecosur, Mexico City, 115-124. 
Chío RE, Guzmán G (1982) Los hongos de la península de Yucatán I las especies de macromicetos conocidas. Biotica 7: 385-398.

de la Fuente JI, Ayala-Vásquez O, Garza-Ocañas F, López CY, García-Jiménez J (2018a) Some interesting Boletales (Basidiomycota) from the state of Quintana Roo, Mexico. Scientia Fungorum 48: 77-86.

de la Fuente JI, Guevara-Guerrero G, López CY, García-Jiménes J (2018b) First record of Mayamontana coccolobae (Stephanosporaceae: Agaricales) from Mexico. Studies in Fungi 3: 34-38. https://doi.org/10.5943/sif/3/1/5

de la Fuente JI, Guevara-Guerrero G, Oros-Ortega I, Sánchez-Zavalegui R, Córdoba-Lara I, García-Jiménez J (2019) Stephanospora mayana (Stephanosporaceae, Russulales), a new sequestrate fungus from Yucatán Peninsula, Mexico. Mycokeys 48: 115-124. https://doi.org/10.3897/mycokeys.48.31007

de la Fuente JI, Hernández-Del Valle JF, Aguirre-Acosta CE, GarcíaJiménez J (2018c) First record of Leucoagaricus lilaceus (Agaricales: Agaricomycetes) from Mexico. Studies in Fungi 3: 187 191. https://doi.org/10.5943/sif/3/1/19

Dennis R (1979) FungusfFlora of Venezuela and adjacent countries. William Clowes and Sons, London, $531 \mathrm{pp}$.

Denison WC (1967) Central American Pezizales. II. The genus Cookeina. Mycologia 59: 306-317. https://doi.org/10.1080/00275 514.1967.12018421

Ferreira AJ, Cortez VG (2012) Lepiotoid Agaricaceae (Basidiomycota) from São Camilo State Park, Paraná state, Brazil. Mycosphere 3: 962-976. https://doi.org/10.5943/mycosphere/3/6/11

García J, Valenzuela R (2005) Hongos Macromicetos. In: SánchezRamos G, Reyes-Castillo P, Dirzo R (Eds) Historia natural de la Reserva de la Biosfera El Cielo, Tamaulipas, México, Universidad Autónoma de Tamaulipas. México City, 321-337.

García-Jiménez J (1999) Estudio sobre la taxonomía, ecología y distribución de algunos hongos de la familia Boletaceae (Basidiomycetes, Agaricales) de México. Master disertation. Universidad Autónoma de Nuevo León, Linares, 334 pp.

García-Jiménez J, Garza-Ocañas F (2001) Conocimiento de los hongos de la familia Boletaceae en México. Ciencia UANL 4: 336344.

Gonzáles-Ávila P, Villegas-Ríos M, Estrada-Torres A (2013) Especies del género Phaeoclavulina en México. Revista Mexicana de Biodiversidad 83: 91-110. https://doi.org/10.7550/rmb.31606

Guevara-Guerrero G, Jiménez J, Castillo J (1985) Algunos Agaricales del norte de México. Revista Mexicana de Micología 1: 129-188.

Guzmán G (1982) New species of fungi from Yucatán Peninsula. Mycotaxon 16: 249-261

Guzmán G (1983) Los hongos de la península de Yucatán II. Nuevas exploraciones y adiciones micológicas. Biotica 8: 71-87.

Guzmán G (1988) Distribución de los hongos en la región del Caribe y zonas aledañas. Caldasia 15: 103-120.

Guzmán G (1998) Inventoring the fungi of Mexico. Biodiversity and Conservation 7: 369-384

Guzmán G (2003) Los hongos de El Edén Quintana Roo (introducción a la micobiota tropical de México). INECOL-CONABIO, Xalapa, $316 \mathrm{pp}$.

Guzmán G (2004) Los hongos de la península de Yucatán (México) V. Nuevas observaciones y nuevos registros. Revista Mexicana de Micología 18: 7-12.

Guzmán G, Bandala VM, Montoya L (1997) An overview on the tropical fungi from Mexico. In: Janardhanan KK, Rajendran C, Nataranjan K, Hawskworth DH (Eds) Tropical mycology. Oxford \& IBH Publishers., New Delhi, 115-148.

Guzmán G, Cortés-Pérez A, Guzmán-Dávalos L, Ramírez-Guillén F, Sánchez-Jácome MR (2013) An emendation of Scleroderma, new records, and review of the known species in Mexico. Revista Mexicana de Biodiversidad 84: 173-191. https://doi.org/10.7550/ rmb.31979

Guzmán G, Montoya L, Bandala VM (1990) Las especies y formas de Dyctiophora (Fungi, Basidiomycetes, Phallales) en México y observaciones sobre su distribución en América Latina. Acta Botánica Mexicana 9: 1-11.

Guzmán G, Montoya-Bello L, Bandala-Muñoz VM (1988) A new species of Psathyrella (Agaricales, Coprinaceae) from Mexico with discussion of the known species. Brittonia 40: 229-234.

Hembrom ME, Parihar A, Das K (2014) Gyrodontium sacchari (Spreng.) Hjortstam - a new record of wood inhabiting hydnoid fungus from India. Journal on New Biological Reports 3: 71-74.

Ibarra-Manríquez G, Villaseñor JL, Durán-García R (1995) Riqueza de especies y endemismo del componente arbóreo de la Península de Yucatán. Boletín de la sociedad botánica de México 57: 49-77.

Ju YM, Rogers JD, San Martín F (1997) A revision of the genus Daldinia. Mycotaxon 61: 243-293.

Kirk PM, Canon PF, Minter DW, Stalpers JA (2008) Ainsworth \& Bisby's dictionary of the fungi. 10 edition. International Mycological Institute, CAB International, Wallingford, $784 \mathrm{p}$.

Kropp BR (2001) Familiar faces in unfamiliar places: mycorrhizal fungi associated with Caribbean pine. Mycologist 15: 137-140.

Lodge DJ, Ammirati JF, O’Dell TE, Mueller G (2004) Collecting and describing macrofungi. In: Mueller G, Bills G, Foster M (Eds) Biodiversity of fungi: inventory and monitoring methods. Elseviere Academic Press, Burlington, Massachusetts, 128-153.

López A, Martínez D, García J (1981) Adiciones al conocimiento de los Phallales del estado de Veracruz. Boletín de la Sociedad Mexicana de Micología 16: 109-116.

López CY, Guevara-Guerrero G, Alonso-Riverol JI (2011) Hongos macromicetos. In: Pozo C, Armijo N, Calmé S (Eds) Riqueza biológica de Quintana Roo. Gobierno del Estado de Quintana RooPrograma de pequeñas donaciones, Mexico City, 24-29.

López-Guzmán LM, Chacón S, Bautista-Gálvez A (2017) Adiciones al conocimiento de los hongos (Macromicetes) de Chiapas, México. Scientia Fungorum 45: 27-35.

Macario-Sánchez PA, Sánchez LC (2011) Pino tropical. In: Pozo C, Armijo N, Calmé S (Eds) Riqueza biológica de Quintana Roo. Gobierno del Estado de Quintana Roo-Programa de pequeñas donaciones, Mexico city, 52-55.

Medel R (2001) Thuemenella cubispora (Ascomycetes, Xylariaceae), un hongo poco común en México. Acta Botánica Mexicana 54: 25-28.

Medel R (2002) Nuevos registros de Pyrenomycetes (Ascomycetes) en México. Boletín de la Sociedad Botánica de México 70: 79-85.

Medel R, Guzmán G, Chacón S (1999) Especies de macromicetos citadas de México IX., Ascomicetes, parte III: 1983-1996. Acta Botánica Mexicana 46: 57-72.

Miller Jr OK, Lodge DJ, Baroni TJ (2000) New and interesting ectomycorrhizal fungi from Puerto Rico, Mona, and Guana Islands. Mycologia 92: 558-570.

Montolla-Bello (1994) Las especies de Lactarius (Fungi, Basidiomycotina) conocidas en México, contribución al estudio del género. Master disertation, Universidad Nacional Autónoma de México, Mexico City, 185 pp.

Mueller GM, Schmit JP (2007) Fungal biodiversity: what do we know? What can we predict? Biodiversity Conservation 16: 1-5. https://doi.org/10.1007/s10531-006-9117-7

Ortíz-Santana B, Lodge DJ, Baroni TJ, Both EE (2007) Boletes from Belize and Dominican Republic. Fungal Diversity 27: 247-416.

Pegler DN (1983) Agaric flora of the Lesser Antilles. Marston Book Services, London, $721 \mathrm{pp}$.

Pegler DN, Fiard JP (1979) Taxonomy and ecology of Lactarius (Agaricales) in the Lesser Antilles. Kew Bulletin 33: 601-628.

Pérez-Silva E, Herrera T, Esqueda-Valle M (1999) Species of Geastrum (Basidiomycota, Geastraceae) from Mexico. Revista Mexicana de Micología 15: 89-104.

Pérez-Silva E, Herrera T, Valenzuela R (1992) Hongos (Macromicetos) de la Península de Yucatán. In: Navarro LD (Ed.) Diversidad biológica de la Reserva de la Biósfera de Sian Ka'an. CIQROO, Mexico City, 13-22. 
Pfister DH, Quijada L, LoBuglio KF (2020) Geodina (Pezizomycetes: Wynneaceae) has a single widespread species in tropical America. Fungal Systematics and Evolution 5: 131-138. https://doi. org/10.3114/fuse.2020.05.08

Pompa-González A, Aguirre-Acosta CE, Encalada-Oliva AV, De Anda-Jáuregui A, Cifuentes-Blanco J, Valenzuela-Garza R (2011) Los macromicetos del jardín botánico de ECOSUR "Dr. Alfredo Barrera Marín” Puerto Morelos, Quintana Roo. Comisión Nacional para el Conocimiento y Uso de la Biodiversidad, México City, 108 pp.

Ramírez-López I, Villegas-Ríos M, Cano-Santana Z (2012) Diversidad de Agaricomycetes clavarioides en la Estación de Biología de Chamela, Jalisco, México. Revista Mexicana de Biodiversidad 83: 1084-1095. https://doi.org/10.7550/rmb.27700

Raymundo T, Valenzuela R, Bautista-Hernández S, Esqueda M, Cifuentes J, Pacheco L (2013) El género Fuscoporia (Hymenochaetales, Basidiomycota) en México. Revista Mexicana de Biodiversidad 84: 50-69. https://doi.org/10.7550/rmb.31604

Robledo GL, Giorgio EM, Franco CRP, Poppof O, Decock C (2014) Gyrodontium sacchari (Spreng.: Fr.) Hjortstam (Boletales, Basidiomycota) in America: new records and its geographic distribution. Check List 10: 1514-1519. https://doi.org/10.15560/10.6.1514

Rodríguez O (2013) El género Pluteus (Agaricales, Pluteaceae) en México. Revista Mexicana de Biodiversidad 84: 129-131. https:// doi.org/10.7550/rmb.31610

Rother MS, da Silveira RMB (2009) Leucoagaricus lilaceus (Agaricaceae), a poorly known Neotropical agaric. Mycotaxon 107: 473-487.

Ryvarden L, Guzmán G (1993) New and interesting polypores from Mexico. Mycotaxon 47: 1-23.

San Martín F, Ju YM, Rogers JD (1999) Algunas especies de Hypoxylon (Pyrenomycetes, Xylariaceae) de México. Acta Botánica Mexicana 47: 31-53.

San Martín F, Rogers JD (1989) A preliminary account of Xylaria from Mexico. Mycotaxon 34: 283-373.

San Martín F, Rogers JD (1993) Kretzschmaria, Leprieuria and Poronia in Mexico. Mycotaxon 47: 179-191.

San Martín F, Rogers JD (1995) Notas sobre la historia, relaciones de hospedante y distribución del género Xylaria (Pyrenomycetes, Sphaeriales) en México. Acta Botánica Mexicana 30: 31-40.

Sánchez-García M, Cifuentes-Blanco J, Matheny BP (2013) Revisión taxonómica del género Melanoleuca en México y descripción de especies nuevas. Revista Mexicana de Biodiversidad 83: 111-127. https://doi.org/10.7550/rmb.31569

Séne S, Avril R, Chaintreuil C, Geoffroy A, Ndiaye C, Diédhiou AG Sadio O, Courtecuisse R, Sylla SN, Selosse MA, Bâ A (2012) Ectomycorrhizal fungal communities of Coccoloba uvifera (L.) L. mature trees and seedlings in the neotropical coastal forests of Guadeloupe (Lesser Antilles). Mycorrhiza 25: 547-549. https:// doi.org/10.1007/s00572-015-0633-8
Sierra S, Cifuentes J (2008) Primer registro del género Cerinomyces (Fungi: Dacrymycetales) en México. Revista Mexicana de Biodiversidad 80: 853-855.

Singer R (1947) The Boletoideae of Florida with notes on extra limital species III. American Midland Naturalist 37: 1-135.

Singer R, Araujo I, Ivory MH (1983) Ectotrophically mycorrizal fungi of the neotropical lowlands, especially central Amazonia. Beihefte zur Nova Hedwigia 77: 7-335.

Singer R, Digilio APL (1951) Pródromo de la Flora Agaricina Argentina. Lilloa 25: 5-461.

Singer R, García J (1988) Two interesting fungi from the Western Hemisphere. The Mycologist 3: 70-71.

Singer R, García J, Gómez LD (1992) The Boletineae of Mexico and Central América IV. Nova Hedwigia 102: 1-99.

Stadler M, Fournier J, Læssøe T, Lechat C, Tichy HV, Piepenbring M (2008) Recognition of hypoxyloid and xylarioid Entonaema species and allied Xylaria species from a comparison of holomorphic morphology, HPLC profiles, and ribosomal DNA sequences. Mycological Progress 7: 53-73. https://doi.org/10.1007/s11557-0080553-5

Tapia F, Chacón S, Pérez-Gorjón S (2016) Hongos Corticioides (Agaricomycetes) citados en México. Acta Botánica Mexicana 114, 1550 .

Torres-Torres MG, Ryvarden L, Guzmán-Dávalos L (2015) Ganoderma subgenus Ganoderma in Mexico. Revista Mexicana de Micología 41: 27-45.

Trierveiler-Pereira L, Meijer AAR, Silveira RMB (2018) Phallales (Agaricomycetes, Fungi) from southern Brazil. Studies in Fungi 4: 162-184. https://doi.org/10.5943/sif/4/1/19

Tun-Cano AA. (2018) Guía de hongos de Cozumel, un mundo por descubrir. Fundación de Parques y Museos de Cozumel, Cozumel, 45 pp.

Valdéz-Hernández M, Islebe GA (2011) Tipos de vegetación en Quintana Roo. In: Pozo C, Armijo N, Calmé S (Eds) Riqueza biológica de Quintana Roo. Gobierno del Estado de Quintana Roo-Programa de pequeñas donaciones, Mexico City, 32-36.

Valenzuela R, Raymundo T, Bautista-Hernández S, Cifuentes J (2013) El género Inonotus s.l. (Hymenochaetales, Agaricomycetes) en México. Revista Mexicana de Biodiversidad 84: 70-90. https:// doi.org/10.7550/rmb.31605

Valenzuela R, Raymundo T, Decock C, Esqueda M (2014) Aphyllophoroid fungi from Sonora, México 2. New records from Sierra de Álamos-Río Cuchujaqui Biosphere Reserve. Mycotaxon 122: 5159. https://doi.org/10.5248/122.51

Villarruel-Ordáz JL, Canseco-Zorrilla E, Cifuentes J (2015) Diversidad fúngica del municipio de San Gabriel Mixtepec, región costa de Oaxaca, México. Revista Mexicana de Micología 41: 55-63.

Weber NS, Smith AH (1985) A field guide to the southern mushrooms. The University of Michigan press, Ann Arbor, Michigan, $280 \mathrm{pp}$. 